\title{
Survivability in Hierarchical Telecommunications Networks
}

\author{
Pierre Fouilhoux \\ Laboratoire LIP6, Université Pierre et Marie Curie, 4 place Jussieu 75005 Paris, France \\ Oya Ekin Karasan \\ Department of Industrial Engineering, Bilkent University, Bilkent, 06800 Ankara, Turkey
}

\author{
A. Ridha Mahjoub \\ LAMSADE, Université Paris-Dauphine, Place du Maréchal de Lattre de Tassigny, 75775 Paris, Cedex 16, France
}

Onur Özkök, and Hande Yaman

Department of Industrial Engineering, Bilkent University, Bilkent, 06800 Ankara, Turkey

\begin{abstract}
The survivable hierarchical telecommunications network design problem consists of locating concentrators, assigning user nodes to concentrators, and linking concentrators in a reliable backbone network. In this article, we study this problem when the backbone is 2-edge connected and when user nodes are linked to concentrators by a point-to-point access network. We formulate this problem as an integer linear program and present a facial study of the associated polytope. We describe valid inequalities and give sufficient conditions for these inequalities to be facet defining. We investigate the computational complexity of the corresponding separation problems. We propose some reduction operations to speed up the separation procedures. Finally, we devise a branch-and-cut algorithm based on these results and present the outcome of a computational study. (c) 2011 Wiley Periodicals, Inc. NETWORKS, Vol. 59(1), 37-58 2012
\end{abstract}

Keywords: Hierarchical network; survivability; separation; facet; branch-and-cut; reduction operation

Received January 2009; accepted January 2010

Correspondence to: A. Ridha Mahjoub; e-mail: mahjoub@lamsade. dauphine.fr

Contract grant sponsor: TUBITAK; Contract grant numbers: 105M322, $107 \mathrm{M} 247$

Contract grant sponsor: CNRS Project BOSPHORE; Contract grant number: 10843 TD

Contract grant sponsor: CGRI-FNRS-CNRS; Contract grant number: 03/005 DOI 10.1002/net.20479

Published online 25 November 2011 in Wiley Online Library (wileyonlinelibrary.com).

(C) 2011 Wiley Periodicals, Inc.

\section{INTRODUCTION}

Network design problems arising in telecommunications applications have originated new challenges in the field of optimization. Within the scope of this study is a two-layer telecommunication network infrastructure. In such a network, the traffic originating from the terminals (user nodes) is communicated through access networks to concentrators (switches or multiplexers) interconnected by a backbone (core) network. The traffic then traverses the backbone network and finally reaches the access network of its destination terminal through multiplexing at the backbone (the interested reader is referred to the surveys of [12] and [20] and the references therein). In the terminology of the hub location literature, the backbone network, carrying large volume of traffic at high speeds, takes the role of the hub network which enables the communication of the terminals or the demand centers. Because the backbone network is the primary means of providing communication between end-users, a reliable topological design is essential. Klincewicz [20] provides a classification of the underlying network design problems based on the topology of the access and the backbone networks. Typical access network topologies include stars, trees, and rings. Similarly, the backbone network may be a star, tree, complete, mesh, or ring.

This study focuses on designs where the access networks are stars and the backbone network is 2-edge connected. A graph is called $k$-edge connected for a non-negative integer $k$ if it contains between any pair of distinct nodes at least $k$ paths that do not share any edge (edge-disjoint). Figure 1 depicts a two level network with a 2-edge connected backbone network 


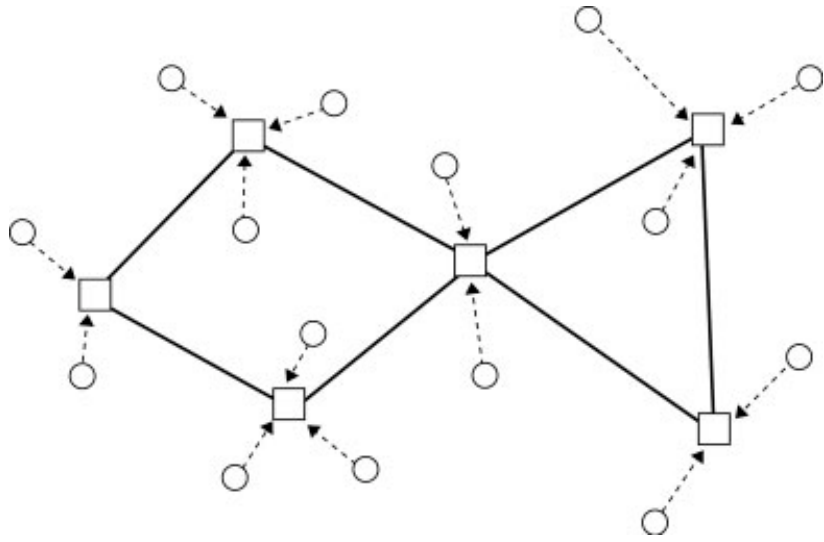

FIG. 1. An example of a 2-edge connected/star network.

and star access networks; here, the squares represent the concentrators and the circles represent the terminals.

This article introduces an in depth analysis of the 2edge connected star subgraph problem (2ECSSP for short) to the telecommunications network design literature. In particular, we seek the most cost effective way of designing a backbone survivable telecommunications network by partitioning a given set of nodes into terminals and concentrators and establishing edges linking concentrators such that each terminal gets assigned to a single concentrator and the edges connecting the concentrators forming the backbone network becomes 2-edge connected.

Our problem inherently has two subproblems, namely the survivable network design problem and the concentrator location problem. Individually, both problems have been widely studied in the literature. Klincewicz [20] presents a survey for such problems. In his classification terminology of backbone network structure/access network structure, 2ECSSP becomes a 2-edge connected/star network design problem. Gourdin et al. [12] review the studies within the telecommunications context which include many variations of the concentrator location problems. Labbé et al. [23] consider the fully connected/star network design problem. Pirkul and Nagarajan [28] and Lee et al. [24] analyze the tree/star network design problem, whereas Gavish [11] studies the star/tree variant. Chardaire et al. [4] analyze the star/star network design problem. A path/path network design problem studied by Current and Pirkul [5] is another example of the two level network design approaches existing in the literature. However, except for the fully connected/star topology design, none of these designs guarantee survivability.

Survivable network design problems have been extensively studied; see for example [14, 16-18,30] for surveys. In particular, the 2-edge connected subgraph problem, which is of close interest to 2ECSSP, is thoroughly investigated in the literature. Studies including [14, 17, 18, 25, 30,31] but not restricted to this list consider the design of 2-edge connected survivable networks. Mahjoub [25] studies the polytope associated with the 2-edge connected subgraph problem, investigates its facets and shows that the polytope is completely described by the trivial and cut inequalities when the underlying graph is series-parallel. Barahona and Mahjoub [2] consider the 2-edge and 2-node connected subgraph problems on Halin graphs and provide the complete descriptions of the associated polytopes. If the 2-edge connected subgraph polytope is completely described by the cut and trivial inequalities for a graph, then the graph is called perfectly 2-edge connected and the problem can be solved in polynomial time. Mahjoub [26] introduces new classes for such graphs and provides sufficient conditions for a graph to be perfectly 2-edge connected. Fonlupt and Mahjoub [7] introduce and study the concept of critical extreme points for the 2-edge connected subgraph polytope. In particular, they characterize the perfectly 2-edge connected graphs.

Several extensions of the 2-edge connected subgraph problem have been investigated in the literature. Kerivin et al. [19] consider the $(1,2)$-survivable network design problem. Here each node is assigned a connectivity type 1 or 2 , and the problem is to determine a subgraph such that between every pair of nodes $(s, t)$, there are at least $\min \{r(s), r(t)\}$ edge-disjoint paths. This is a special case of a more general model introduced by Grötschel et al. [14]. Vandenbussche and Nemhauser [31] study the 2-edge connected subgraph problem on graphs when an edge may be used more than once. Fortz et al. [8,9] study the problem of designing 2-nodeconnected networks with bounded meshes. The network to be designed is 2-node connected and every edge of the network must be contained in a cycle whose length is bounded from above. They describe several classes of valid inequalities and propose a branch-and-cut algorithm. Fortz et al. [10] study a similar problem in which the network to be designed is 2-edge connected. Further work on the $k$-edge connected subgraph problem with (and without) hop constraints can be found in $[3,6,16]$.

Labbé et al. [21] study the ring/star network design problem which is of close kinship to the 2ECSSP. Indeed, this problem is a restriction of the 2ECSSP as a ring is a 2-edge connected network. The authors identify some facet defining inequalities of the associated polytope. A branch-and-cut algorithm is developed based on these inequalities and computational results are presented. We note here that as 2ECSSP is a relaxation of the ring/star network design problem, any valid inequality for the 2ECSSP polytope is also valid for the ring/star polytope; however, the converse is not necessarily true.

This work contributes to the two level network design literature by expanding it with the 2ECSSP, a special survivable infrastructure that has not yet been analyzed in the literature. The contribution includes a $0-1$ model development and a detailed polyhedral analysis of the associated polytope. For the families of facet defining inequalities, the computational complexity status of each separation problem is established and exact and/or heuristic separation algorithms are designed. To speed up the separation runtime, some reduction operations are proposed and analyzed. Finally, a branch-and-cut algorithm which assembles all this theoretical development is designed for the 2ECSSP. 
This article is organized as follows. In the following section, we give an integer programming formulation for the 2ECSSP. In Section 3, the polytope associated with 2ECSSP is analyzed and several classes of valid inequalities for the 2ECSSP are derived. We give necessary and sufficient conditions for these inequalities to be facet defining. The reduction operations are proposed in Section 4, and the separation algorithms are discussed in Section 5. In Section 6, we present the computational study and finally we give some concluding remarks in Section 7.

\section{NOTATION AND MATHEMATICAL MODEL}

We first give some notation. We consider directed and undirected graphs. We denote an undirected graph by $G=$ $(V, E)$ where $V$ is the node set and $E$ is the edge set of $G$. If $e \in E$ is an edge between two nodes $i$ and $j$, then we also write $e=i j$ or $e=\{i, j\}$ to denote $e$. If $V_{1}$ and $V_{2}$ are two node subsets such that $V_{1} \cap V_{2}=\emptyset$, then we denote by $\left[V_{1}, V_{2}\right]$ the set of edges having one node in $V_{1}$ and the other in $V_{2}$. Given a set $S \subseteq V$, we let $\delta_{G}(S)=[S, V \backslash S]$, that is the set of edges having exactly one node in $S$. We will omit the subscript if the context is clear. The edge set $\delta(S)$ is called a cut. For $i \in V$, we will write $\delta(i)$ instead of $\delta(\{i\})$.

A directed graph will be denoted by $D=(V, A)$ where $V$ is the node set and $A$ is the arc set. If $a \in A$ is an arc from node $i$ to node $j$, then we also write $a=(i, j)$ to denote $a$. For $S \subseteq V$, we let $G(S)(D(S))$ denote the subgraph of $G(D)$ induced by $S$, that is the subgraph whose node set is $S$ and edge (arc) set is $E(S)(A(S))$, the set of edges (arcs) in $G(D)$ having both nodes in $S$.

Given a vector $x \in \mathbb{R}^{|E|}$ and $F \subseteq E$, we let $x(F)=$ $\sum_{e \in F} x_{e}$

We now proceed with a formal description of the 2ECSSP to be followed with a $0-1$ model. We assume that $V=$ $\{0,1, \ldots, n\}$ is a given set of terminals. Node 0 is a special concentrator corresponding to the root node in the two level network infrastructure. Let $E=\{\{i, j\}: i \in V, j \in V \backslash\{i\}\}$ represent the set of potential backbone links. Thus we assume a complete graph in terms of edges. Associated with installing a backbone link $e \in E$ is a non-negative fixed setup cost $c_{e}$. Similarly, there is a non-negative assignment cost of $d_{i j}$ units associated with assigning terminal $i \in V$ to concentrator $j \in V$. In particular, $d_{i i}$ corresponds to the cost of installing a concentrator at node $i \in V$. Note that $d_{i j}$ and $d_{j i}$ might be different.

Given $V, 2 \mathrm{ECSSP}$ seeks a partition of $V$ into $C$ and $T$ such that $0 \in C$, a set of backbone links $E^{\prime} \subseteq E$ between nodes in $C$ such that the graph $\left(C, E^{\prime}\right)$ is 2-edge connected, and an assignment of each node in $T$ to one in $C$ such that the total cost of installing backbone links and concentrators and assigning terminals to concentrators is minimum. 2ECSSP is NP-hard since it possesses as a special case the 2-edge connected subgraph problem, which is NP-hard [26].

Against this background, we define $x_{e}$ to be 1 if edge $e \in E$ is used in the backbone network and 0 otherwise and $y_{i j}$ to be 1 if node $i \in V$ is assigned to node $j \in V$ and 0 otherwise. If a concentrator is installed at node $i \in V$ then node $i$ is assigned to itself, i.e., $y_{i i}=1$.

Using these two sets of binary variables, we can model the 2ECSSP as follows:

$$
\begin{aligned}
& z=\min \sum_{e \in E} c_{e} x_{e}+\sum_{i \in V} \sum_{j \in V} d_{i j} y_{i j} \\
& \text { s.t. } \sum_{j \in V} y_{i j}=1 \quad \forall i \in V, \\
& y_{i j}+x_{i j} \leq y_{j j} \quad \forall i, j \in V, i \neq j, \\
& y_{00}=1 \\
& x(\delta(S)) \geq 2 \sum_{j \in S} y_{i j} \quad \forall S \subseteq V \backslash\{0\}, i \in S, \\
& x_{e} \in\{0,1\} \quad \forall e \in E, \\
& y_{i j} \in\{0,1\} \quad \forall i, j \in V .
\end{aligned}
$$

Constraints (2) and (3) ensure that either a concentrator is installed at a given node or this node is assigned to exactly one other node where a concentrator is installed. If an edge becomes a backbone edge, then concentrators are installed at both endpoints of this edge due to constraints (3). Constraint (4) fixes the value of $y_{00}$ to one and hence a concentrator is installed at the root node 0 . Constraints (5) ensure 2-edge connectivity in the backbone network. Consider a node subset $S \subseteq V \backslash\{0\}$ and a node $i \in S$. If $i$ is assigned to some node in set $S$, i.e., if $\sum_{j \in S} y_{i j}=1$, then there is at least one concentrator, say $k$ in $S$, implying that $i$ and $k$ must be linked by at least two edge-disjoint paths, and hence at least two edges from $\delta(S)$ have to be included in the backbone network. Finally, the objective function is the sum of the cost of installing the backbone edges and the concentrators and that of assigning the remaining nodes to concentrators.

2ECSSP is a relaxation of the ring/star network design problem obtained by dropping the requirement that each concentrator is adjacent to exactly two backbone edges. The formulation above is obtained by removing the degree constraints from the formulation of the ring/star network design problem given in Labbé et al. [21].

The model above is based on the assumption of the existence of a root node that is a hub. This root node might be a central unit to which other concentrators should be connected or it might be desired to connect the backbone network to an already existing higher level network at this point. In such cases, the existence assumption of a root node is reasonable. If there is no such node, then constraint (5) can be modified as follows:

$$
\begin{aligned}
x(\delta(S))+2 \sum_{j \in V \backslash S} y_{i j}+2 \sum_{j \in S} y_{k j} & \\
& \geq 2 \quad \forall S \subset V, \forall i \in V, \forall k \in V \backslash\{i\}
\end{aligned}
$$

These constraints force the model to install at least two edges between sets $S$ and $V \backslash S$ if at least one concentrator is installed in each set. 


\section{POLYHEDRAL ANALYSIS}

In this section, we present a polyhedral analysis for the convex hull of the solutions to the 2ECSSP. We first project out some variables to make the analysis easier. From constraints (2), for $i \in V \backslash\{0\}$, we can eliminate variable $y_{i i}$ by substituting $y_{i i}=1-\sum_{j \in V \backslash\{i\}} y_{i j}$. Additionally, the variables related to the assignment of the root node can also be dropped because their values are known $\left(y_{00}=1\right.$ and $y_{0 i}=0$ for all $i \in V \backslash\{0\}$ ). After substitution, constraint (3) becomes $x_{i j}+y_{i j}+\sum_{k \in V \backslash\{j\}} y_{j k} \leq 1$ for a given node pair $i, j$. For the node pair $0, i$ we obtain the constraint $x_{0 i}+$ $y_{0 i}+\sum_{k \in V \backslash\{i\}} y_{i k} \leq 1$, whereas $i, 0$ yields $x_{0 i}+y_{i 0}+$ $\sum_{k \in V \backslash\{0\}} y_{0 k} \leq 1$. Because $y_{00}=1$ and $y_{0 i}=0$ for all $i \in V \backslash\{0\}$, these two constraints become $x_{0 i}+\sum_{k \in V \backslash\{i\}} y_{i k} \leq$ 1 and $x_{0 i}+y_{i 0} \leq 1$, respectively. Clearly, the first one dominates the latter, so we only use the first inequality. Let $A=\{(i, j): i \in V \backslash\{0\}, j \in V \backslash\{i\}\}$ and define $d_{i j}^{\prime}=d_{i j}-d_{i i}$ for each $(i, j) \in A$. Now we obtain the following equivalent formulation:

$$
\begin{array}{cl}
z=\sum_{i \in V} d_{i i}+\min \sum_{e \in E} c_{e} x_{e}+\sum_{(i, j) \in A} d_{i j}^{\prime} y_{i j} \\
\text { s.t. } x_{i j}+y_{i j}+\sum_{k \in V \backslash\{j\}} y_{j k} \leq 1 & \forall(i, j) \in A: j \neq 0 \\
x_{0 i}+\sum_{k \in V \backslash\{i\}} y_{i k} \leq 1 & \forall i \in V \backslash\{0\} \\
x(\delta(S))+2 \sum_{j \in V \backslash S} y_{i j} \geq 2 & \forall S \subseteq V \backslash\{0\}, i \in S \\
0 \leq x_{e} \leq 1 & \\
0 \leq y_{i j} \leq 1 & \forall e \in E \\
x_{e} \text { integer } & \forall(i, j) \in A . \\
y_{i j} \text { integer } & \forall e \in E
\end{array}
$$

Inequalities (10) will be called cut inequalities and inequalities (11)-(12) are called trivial inequalities. Let $X=\left\{(x, y) \in R^{|E|+|A|}:(x, y)\right.$ satisfies (8)-(14) $\}$ and $\mathcal{P}=$ $\operatorname{conv}(X)$. The remaining part of this section is devoted to the analysis of facets of the polytope $\mathcal{P}$. We show that the constraints used in the model define facets of $\mathcal{P}$. In doing this, we investigate the relationship between some facets of a special stable set polytope and the facets of $\mathcal{P}$. Then, we extend a known family of facet defining inequalities for the 2-edge connected subgraph polytope to be valid for our model, and prove that they are facet defining for $\mathcal{P}$ under some conditions. Finally, we propose a new family of facet defining inequalities.

To this end, we first introduce some more notation. For $e \in E$, let $\chi_{e}$ be a unit vector of size $|E|$ with the entry corresponding to edge $e$ equal to 1 and other entries equal to 0 . Similarly, for $(i, j) \in A$, let $\gamma_{i j}$ be a unit vector of size $|A|$ with the entry corresponding to arc $(i, j)$ equal to 1 and other entries equal to 0 . Hence, for a vector $x \in\{0,1\}^{|E|}$ (resp. $\left.y \in\{0,1\}^{|A|}\right)$, if $F$ is the set of edges $e$ (resp. $\operatorname{arcs} a$ ) such that $x_{e}=1$ (resp. $\left.y_{a}=1\right)$, then $x$ (resp. $y$ ) can also be written as $\sum_{e \in F} \chi_{e}$ (resp. $\left.\sum_{a \in F} \gamma_{a}\right)$.

In the sequel, we assume that $|V| \geq 5$. If $|V|<5$, then the problem is easy to solve. Moreover, some of the inequalities of the formulation do not define facets of the associated polytope.

\subsection{Dimension and Trivial Facets}

We first investigate the dimension of $\mathcal{P}$ and study its trivial facet defining inequalities.

Theorem 1. $\mathcal{P}$ is full dimensional.

Proof. Consider the solutions $\left(\sum_{e \in E} \chi_{e}, 0\right)$, $\left(\sum_{e \in E \backslash\left\{e^{\prime}\right\}} \chi_{e}, 0\right)$ for $e^{\prime} \in E$ and $\left(\sum_{e \in E \backslash \delta(i)} \chi_{e}, \gamma_{i j}\right)$ for $(i, j) \in A$. They are in $\mathcal{P}$ and are affinely independent. Hence $\operatorname{dim}(\mathcal{P})=|E|+|A|$.

Theorem 2. For $e \in E$, inequality $x_{e} \geq 0$ is facet defining for $\mathcal{P}$.

Proof. Let $\mathcal{F}=\left\{(x, y) \in \mathcal{P}: x_{e}=0\right\}$. The solutions $\left(\sum_{e^{\prime} \in E \backslash\{e\}} \chi_{e^{\prime}}, 0\right),\left(\sum_{e^{\prime \prime} \in E \backslash\left\{e, e^{\prime}\right\}} \chi_{e^{\prime \prime}}, 0\right)$ for $e^{\prime} \in E \backslash\{e\}$, $\left(\sum_{e^{\prime} \in E \backslash \delta(k)} \chi_{e^{\prime}}, \gamma_{k l}\right)$ for $(k, l) \in A$ with $k \in e$ ( $k$ is an endpoint of $e$ ), and $\left(\sum_{e^{\prime} \in E \backslash(\delta(k) \cup\{e\})} \chi_{e^{\prime}}, \gamma_{k l}\right)$ for $(k, l) \in A$ with $k \notin e$ ( $k$ is not an endpoint of $e$ ), constitute a family of $|E|+|A|$ affinely independent solutions in $\mathcal{F}$.

Theorem 3. For $(i, j) \in A$, inequality $y_{i j} \geq 0$ is facet defining for $\mathcal{P}$.

Proof. Let $\mathcal{F}=\left\{(x, y) \in \mathcal{P}: y_{i j}=0\right\}$. The solutions $\left(\sum_{e \in E} \chi_{e}, 0\right),\left(\sum_{e \in E \backslash\left\{e^{\prime}\right\}} \chi_{e}, 0\right)$ for $e^{\prime} \in E$ and $\left(\sum_{e \in E \backslash \delta(k)} \chi_{e}, \gamma_{k l}\right)$ for $(k, l) \in A \backslash\{(i, j)\}$ are in $\mathcal{F}$ and are affinely independent.

Inequalities $x_{i j} \leq 1$ and $y_{i j} \leq 1$ are not facet defining as they are implied by constraints (8) and (9).

\subsection{Cut Inequalities}

After establishing the dimension of $\mathcal{P}$ and its trivial facet defining inequalities, we focus on the cut inequalities (10). In the following theorem, we give necessary and sufficient conditions for these inequalities to be facet defining for $\mathcal{P}$.

Theorem 4. Let $S \subseteq V \backslash\{0\}$ such that $S \neq \emptyset$ and $i \in S$. Inequality (10) defines a facet of $\mathcal{P}$ if and only if $|S| \neq 2$ and $|V \backslash S| \neq 2$.

Proof. Suppose that $|S|=2$ and $S=\{i, j\}$. Then inequality (10) for this choice of $S$ and $i$ is

$$
x(\delta(\{i, j\}))+2 \sum_{k \in V \backslash\{i, j\}} y_{i k} \geq 2 .
$$


Summing the cut inequalities (10) for $S=\{i\}$ and $S=\{j\}$ yields $x(\delta(i))+x(\delta(j))+2 \sum_{k \in V \backslash\{i\}} y_{i k}+2 \sum_{k \in V \backslash\{j\}} y_{j k} \geq 4$. Substituting $x(\delta(i))+x(\delta(j))=x(\delta(\{i, j\}))+2 x_{i j}$ and adding constraint (8), $-2 x_{i j}-2 y_{i j}-2 \sum_{k \in V \backslash\{j\}} y_{j k} \geq-2$, we obtain inequality (15), and hence (15) is not facet defining.

Now suppose that $V \backslash S=\{0, j\}$ and $j \in V \backslash\{0, i\}$. Let $(x, y) \in X$ be a solution which satisfies the corresponding cut inequality (10) at equality. If $x_{0 j}=1$, because $x$ induces a 2edge connected subgraph, we should have $\sum_{k \in S} x_{0 k} \geq 1$ and $\sum_{k \in S} x_{j k} \geq 1$. As inequality (10) for $S=V \backslash\{0, j\}$ is tight for $(x, y)$, it thus follows that $\sum_{k \in S} x_{0 k}=1$ (and $\sum_{k \in S} x_{j k}=1$ ). If $x_{0 j}=0$, then one should have $\sum_{k \in S} x_{0 k}=2$, and therefore $\sum_{k \in S} x_{j k}=0$. In both cases $(x, y)$ satisfies $x_{0 j}-\sum_{k \in S} x_{j k}=$ 0 . As this equation is not a multiple of $x(\delta(V \backslash\{0, j\}))+2\left(y_{i 0}+\right.$ $\left.y_{i j}\right)=2$, inequality (10) is not facet defining.

Now suppose that $|S|>2$ and $|V \backslash S|>2$. Notice that as $G$ is complete, $G(S)$ and $G(V \backslash S)$ are 2-edge connected. Let $\mathcal{F}=$ $\left\{(x, y) \in \mathcal{P}: x(\delta(S))+2 \sum_{j \in V \backslash S} y_{i j}=2\right\}$. Suppose that every solution $(x, y)$ in $\mathcal{F}$ also satisfies $a x+b y=\beta$. We will show that $a x+b y=\beta$ is a multiple of $x(\delta(S))+2 \sum_{j \in V \backslash S} y_{i j}=2$.

Consider solution $(x, 0)$ where $x=\sum_{e \in E(S) \cup E(V \backslash S)} \chi_{e}+$ $\chi e_{1}+\chi_{e_{2}}$ and $e_{1}$ and $e_{2}$ are any two edges in $\delta(S)$. Let $e^{\prime} \in$ $\delta(S) \backslash\left\{e_{1}, e_{2}\right\}$. As $(x, 0)$ and the solutions $\left(x+\chi_{e^{\prime}}-\chi_{e_{1}}, 0\right)$ and $\left(x+\chi_{e^{\prime}}-\chi_{e_{2}}, 0\right)$ are both in $\mathcal{F}$, we have $a_{e_{1}}=a_{e_{2}}=a_{e^{\prime}}$. Therefore $a_{e^{\prime}}=\sigma$ for all $e^{\prime} \in \delta(S)$ for some $\sigma \in \mathbb{R}$.

Let $e^{\prime} \in E(S)$ and let $e_{1}, e_{2}$ be two edges in $\delta(S)$ incident to the two endpoints of $e^{\prime}$ such that $e_{1} \cap e_{2} \cap e^{\prime}=\emptyset$. Consider the solution $(x, 0)$ where $x=\sum_{e \in E(S) \cup E(V \backslash S)} \chi_{e}+\chi_{e_{1}}+\chi_{e_{2}}$. As $(x, 0)$ and the solution $\left(x-\chi_{e^{\prime}}, 0\right)$ are both in $\mathcal{F}$, we have $a_{e^{\prime}}=0$. We can show similarly that $a_{e^{\prime}}=0$ for all $e^{\prime} \in E(V \backslash S)$.

Let $j \in V \backslash\{i, 0\}$ and let $e_{1}, e_{2}$ be two edges in $\delta(S) \backslash \delta(j)$ with different endpoints in $S$ if $j \in S$ and with different endpoints in $V \backslash S$ if $j \in V \backslash S$. Consider $(x, 0)$ where $x=\sum_{e \in E(S) \cup E(V \backslash S)} \chi_{e}+\chi_{e_{1}}+\chi_{e_{2}}$. This solution is in $\mathcal{F}$. Observe that, $\left(x-\sum_{e \in \delta(j)} x_{e} \chi_{e}, \gamma_{j k}\right)$ is also in $\mathcal{F}$ for any $k \in V \backslash\{j\}$. As $a_{e}=0$ for all $e \in E(S) \cup E(V \backslash S)$ we have $b_{j k}=0$.

Similarly, the solution $(x, 0)$ where $x=\sum_{e \in E(S) \cup E(V \backslash S)} \chi_{e}$ $+\chi_{e_{1}}+\chi_{e_{2}}$ and $e_{1}$ and $e_{2}$ are two edges in $\delta(S) \backslash \delta(i)$ is in $\mathcal{F}$. Let $k \in S \backslash\{i\}$. As the solution $\left(x-\sum_{e \in \delta(i)} x_{e} \chi_{e}, \gamma_{i k}\right)$ is also in $\mathcal{F}$, we have $b_{i k}=0$.

Let $j \in V \backslash S$ and consider $(x, 0)$ where $x=$ $\sum_{e \in E(S) \cup E(V \backslash S)} \chi_{e}+\chi_{e_{1}}+\chi_{e_{2}}$ and $e_{1}$ and $e_{2}$ are two edges in $\delta(S)$. As $\left(x-\sum_{e \in E(S)} \chi_{e}-\chi_{e_{1}}-\chi_{e_{2}}, \sum_{k \in S} \gamma_{k j}\right)$ is in $\mathcal{F}$, $b_{k j}=0$ for every $k \neq i, a_{e}=0$ for all $e \in E(S)$, and $a_{e_{1}}=a_{e_{2}}=\sigma$, we have $b_{i j}=2 \sigma$.

If $|S|=1$ or $|V \backslash S|=1$, computation of $a$ and $b$ is almost the same. The difference is that if $|S|=1$, then $E(S)=\emptyset$, there is not a node $j \in S \backslash\{i\}$ and there is not an arc $(i, j)$ with $j \in S$ as $S=\{i\}$. Similarly, if $|V \backslash S|=1$, then $E(V \backslash S)=\emptyset$ and there is not a node $j \in V \backslash(S \cup\{0\})$. So we do not calculate the corresponding coefficients. Computation of other coefficients is still valid.

Hence, $a x+b y=\beta$ is a multiple of $x(\delta(S))+$ $2 \sum_{j \in V \backslash S} y_{i j}=2$ and $\mathcal{F}$ is a facet of $\mathcal{P}$.

\subsection{Stable Set Relaxation and Clique Inequalities}

Let $X_{S}=\left\{(x, y) \in R^{|E|+|A|}:(x, y)\right.$ satisfies (8), (9), (13), and (14) $\}$ and $\mathcal{P}_{\mathcal{S}}=\operatorname{conv}\left(X_{S}\right)$. The polytope $\mathcal{P}_{\mathcal{S}}$ is a stable set polytope. As $\mathcal{P} \subseteq \mathcal{P}_{\mathcal{S}}$ and $\mathcal{P}$ is full dimensional, $\mathcal{P}_{\mathcal{S}}$ is also full dimensional. Let $\alpha x+\beta y \leq \beta_{0}$ be a facet defining inequality for $\mathcal{P}$. If this inequality is valid for $\mathcal{P}_{\mathcal{S}}$, then it also defines a facet of $\mathcal{P}_{\mathcal{S}}$. This implies that the inequalities $x_{e} \geq 0$ for $e \in E$ and $y_{i j} \geq 0$ for $(i, j) \in A$ are facet defining for $\mathcal{P}_{\mathcal{S}}$. The trivial inequalities $x_{e} \leq 1$ for $e \in E$ and $y_{i j} \leq 1$ for $(i, j) \in A$ are implied by constraints (8) and (9) and hence do not define facets of $\mathcal{P}_{\mathcal{S}}$. Moreover, as $X_{S}$ is an independence system, if $\alpha x+\beta y \leq \beta_{0}$ is a nontrivial facet defining inequality for $\mathcal{P}_{\mathcal{S}}$, then $\alpha \geq 0, \beta \geq 0$, and $\beta_{0}>0$.

In the following two theorems, we investigate how some of the facets of $\mathcal{P}_{\mathcal{S}}$ are related to those of $\mathcal{P}$.

Theorem 5. Let $e=\{i, j\} \in E$ with $i \neq 0$ and $j \neq 0$. Suppose that inequality $\alpha_{e} x_{e}+\beta y \leq \beta_{0}$ is a nontrivial facet defining inequality for $\mathcal{P}_{\mathcal{S}}$. If

i. for all $m \in V \backslash\{0, i, j\}$ and $l \in V \backslash\{0, i, j, m\}$, there exists a node $k \in V \backslash\{0, m, l\}$ such that $\beta_{k m}=\beta_{k l}=\beta_{k 0}=0$,

ii. for $m \in V \backslash\{0, i, j\}$, there exists a node $k \in V \backslash\{0, i, j, m\}$ such that $\beta_{k m}=\beta_{k i}=\beta_{k j}=\beta_{k 0}=0$,

iii. for $m \in V \backslash\{0, i, j\}$, there exist two distinct nodes $k_{1}, k_{2} \in$ $V \backslash\{0, m\}$ such that $\beta_{k_{1} m}=\beta_{k_{1} 0}=\beta_{k_{2} m}=\beta_{k_{2} 0}=0$ and $\left|\left\{k_{1}, k_{2}\right\} \cap\{i, j\}\right| \leq 1$,

$i v$. there exist two distinct nodes $k_{1}, k_{2} \in V \backslash\{0, i, j\}$ such that $\beta_{k_{1} 0}=\beta_{k_{1} i}=\beta_{k_{1} j}=\beta_{k_{2} 0}=\beta_{k_{2} i}=\beta_{k_{2} j}=0$,

are all satisfied, then the inequality $\alpha_{e} x_{e}+\beta y \leq \beta_{0}$ also defines a facet of $\mathcal{P}$.

Proof. See the appendix.

Theorem 6. Let $e=\{0, i\} \in$ E. Suppose that the inequality $\alpha_{e} x_{e}+\beta y \leq \beta_{0}$ is facet defining for $\mathcal{P}_{\mathcal{S}}$. If

i. for all $m \in V \backslash\{0, i\}$ and $l \in V \backslash\{0, i, m\}$, there exists a node $k \in V \backslash\{0, i, m, l\}$ such that $\beta_{k i}=\beta_{k m}=\beta_{k l}=\beta_{k 0}=0$,

ii. for $m \in V \backslash\{0, i\}$, there exist two distinct nodes $k_{1}, k_{2} \in$ $V \backslash\{0, i, m\}$ such that $\beta_{k_{1} m}=\beta_{k_{1} i}=\beta_{k_{1} 0}=\beta_{k_{2} m}=\beta_{k_{2} i}=$ $\beta_{k_{2} 0}=0$,

are all satisfied, then the inequality $\alpha_{e} x_{e}+\beta y \leq \beta_{0}$ also defines a facet of $\mathcal{P}$.

Proof. Similar to the proof of Theorem 5.

Consider the conflict graph, in which there is an edge between two nodes if these two nodes cannot be found in a solution simultaneously, associated with the set $X_{S}=$ $\left\{(x, y) \in R^{|E|+|A|}:(x, y)\right.$ satisfies (8), (9), (13), and (14) $\}$. It is known that a clique inequality is facet defining for the stable set polytope if and only if the underlying clique is maximal [1]. Now, we investigate the maximal cliques in the conflict graph associated with $X_{S}$. 
Theorem 7. The only facet defining clique inequalities for $\mathcal{P}_{\mathcal{S}}$ are constraints (8) and (9), and inequalities $y_{i j}+y_{j k}+$ $y_{k i} \leq 1$ for distinct nodes $i, j, k \in V \backslash\{0\}$.

Proof. Consider a maximal clique in the conflict graph associated with $X_{S}$. Observe that this clique can contain at most one $x_{e}$ variable. First suppose that $x_{i j}$ is in the clique for some $\{i, j\} \in E$ such that $i \neq 0$ and $j \neq 0$. Then, the neighbors of $x_{i j}$ are nodes of the form $y_{i l}$ for $l \in V \backslash\{i\}$ and $y_{j k}$ for $k \in V \backslash\{j\}$. Assume without loss of generality that the clique contains a node $y_{i l}$ for some $l \in V \backslash\{i\}$. Then, there exists a node of the form $y_{j k}$ for some $k \in V \backslash\{j\}$ in the clique only if $l=j$. If $l=j$, then the clique contains all nodes of the form $y_{j k}$ for $k \in V \backslash\{j\}$ and no other node. The corresponding clique inequality is (8). Now suppose that the clique does not contain any node of the form $y_{j k}$ for $k \in V \backslash\{j\}$. Then, it contains all nodes $y_{i l}$ for $l \in V \backslash\{i\}$ and no other node. However, such a clique cannot be maximal as it can be enlarged by adding the node $y_{j i}$. Hence, we arrive at a contradiction.

Now suppose that $x_{0 i}$ is in the clique for some $i \in V \backslash\{0\}$. The neighbors of $x_{0 i}$ are nodes of the form $y_{i k}$ for $k \in V \backslash\{i\}$. The node $x_{0 i}$ together with the nodes $y_{i k}$ for all $k \in V \backslash\{i\}$ form a maximal clique and the corresponding clique inequality is (9).

The remaining maximal cliques do not include any node of the form $x_{e}$ for $e \in E$. Suppose that we have such a maximal clique which includes a node $y_{i j}$ for some $(i, j) \in A$. If the clique also includes a node of the form $y_{i l}$ for $l \in V \backslash\{i, j\}$, then it can only include the other nodes $y_{i k}$ for $k \in V \backslash\{i, j, l\}$ and a node $y_{m i}$ for some $m \in V \backslash\{0, i\}$. Such a clique cannot be maximal as it can be extended by adding the node $x_{m i}$. The neighbors of $y_{i j}$ other than those of the form $x_{e}$ for $e \in E$ and $y_{i l}$ for $l \in V \backslash\{i, j\}$ are the nodes $y_{j k}$ for some $k \in V \backslash\{j\}$ if $j \neq$ 0 . If $j=0$, then there is no such neighbor. Suppose that $j \neq 0$ and that the clique contains $y_{i j}$ and $y_{j k}$ for some $k \in V \backslash\{j\}$. If the clique contains another node $y_{j l}$ for $l \in V \backslash\{j, k\}$, then it can only include the other nodes $y_{j m}$ for $m \in V \backslash\{j, k, l\}$ and can be extended by adding the node $x_{i j}$. Hence, if a maximal clique includes nodes $y_{i j}$ and $y_{j k}$ and does not include any node of the form $x_{e}, y_{i l}$ for $l \in V \backslash\{i, j\}$, and $y_{j l}$ for $l \in V \backslash\{j, k\}$, then it should contain the node $y_{k i}$. The associated clique inequality is $y_{i j}+y_{j k}+y_{k i} \leq 1$.

The inequalities of type $y_{i j}+y_{j k}+y_{k i} \leq 1$ are also known as the triangle inequalities. As consequences of Theorems $5-7$, we have the following:

Corollary 1. Let $(i, j) \in A$ with $j \neq 0$. Then, inequality (8) is facet defining for $\mathcal{P}$.

Corollary 2. Let $i \in V \backslash\{0\}$. Then, inequality (9) is facet defining for $\mathcal{P}$.

Corollary 3. Let $i, j$, and $k$ be distinct nodes in $V \backslash\{0\}$. Then, inequality $y_{i j}+y_{j k}+y_{k i} \leq 1$ is facet defining for $\mathcal{P}$.

Corollaries 1 and 2 together with Theorem 4 show that all the constraints of the model are facet defining for the

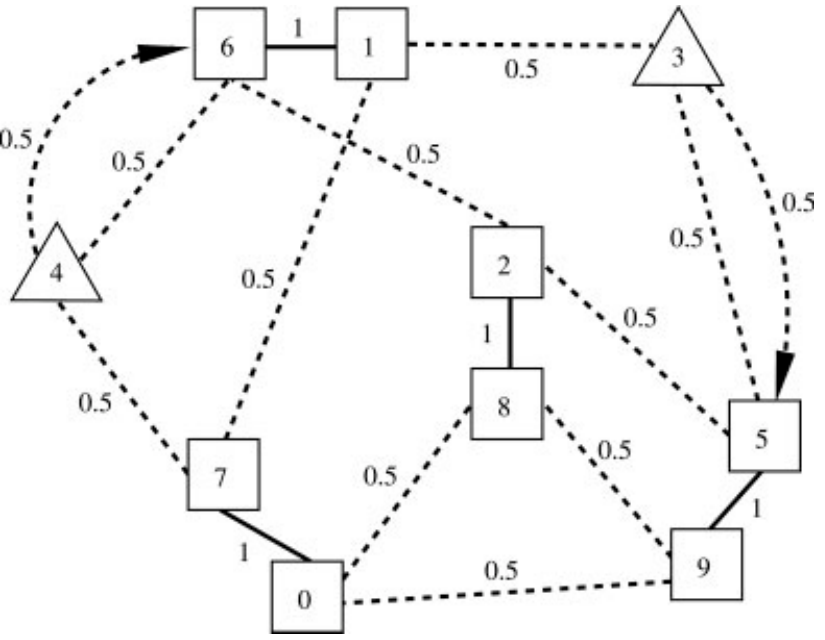

FIG. 2. A fractional solution cut off by an $F$-partition inequality.

polytope $\mathcal{P}$. In the sequel, we present two other families of facet defining inequalities.

\subsection{Extended F-Partition Inequalities}

An important class of valid inequalities for the 2-edge connected subgraph problem is the class of $F$-partition inequalities, which are shown to be very effective for solving large instances of the 2-edge connected subgraph problem (see $[19,25])$. In this section, we extend these inequalities to be valid for the 2ECSSP polytope $\mathcal{P}$, give some sufficient conditions for these inequalities to be facet defining, and investigate the complexity of the associated separation problem.

We first give an example of a fractional solution $(\bar{x}, \bar{y})$ and an extended $F$-partition inequality that cuts off this solution. Consider the solution $(\bar{x}, \bar{y})$ depicted in Figure 2. Let $V=\{0, \ldots, 9\}$. The edges and arcs with value 0 are omitted. The positive values in $(\bar{x}, \bar{y})$ are either 0.5 or 1 . The backbone edges with value 1 are represented by bold lines and those with value 0.5 are represented by dashed lines. The assignments are as follows: $\bar{y}_{i i}=1$ for $i \in V \backslash\{3,4\}$ (these nodes are represented by rectangles) and $\bar{y}_{33}=\bar{y}_{35}=\bar{y}_{44}=\bar{y}_{46}=0.5$ (these nodes are represented by triangles and the assignments of 3 to 5 and 4 to 6 are represented by dashed lines with arrows). The solution $(\bar{x}, \bar{y})$ satisfies all the clique (8) and cut (10) inequalities and is an extreme point of the linear relaxation of 2ECSSP.

Consider a partition of $V$ into $V_{0}, \ldots, V_{5}$ such that $V_{0}=$ $\{0,8,9\}, V_{1}=\{1\}, V_{2}=\{2\}, V_{3}=\{3,5\}, V_{4}=\{4,6\}$, and $V_{5}=\{7\}$. Let $F=\{\{0,7\},\{2,8\},\{5,9\}\}$. Each set in the partition has at least one node at which a concentrator is installed. Hence, to have 2-edge connectedness among the sets of the partition, we need to use at least 4 edges from the set $\delta\left(V_{0}, \ldots, V_{5}\right) \backslash F$. Hence, we need $x\left(\delta\left(V_{0}, \ldots, V_{5}\right) \backslash F\right) \geq 4$. Notice that as the root node is in $V_{0}$, there is a concentrator installed in the set $V_{0}$ in any fractional solution. This is not necessarily true for the remaining sets of the partition. For 
instance if node 1 is assigned to another node, then there is no concentrator installed in set $V_{1}$ and we need 3 edges from the set $\delta\left(V_{0}, \ldots, V_{5}\right) \backslash F$ for 2-edge connectedness. So we need $x\left(\delta\left(V_{0}, \ldots, V_{5}\right) \backslash F\right)+\sum_{j \in V \backslash\{1\}} y_{1 j} \geq 4$. We can repeat the same argument for the remaining sets of the partition. Here for sets that are not singletons, we can only use one node in the inequality. Suppose that we pick node 3 for set $V_{3}$ and node 4 for set $V_{4}$. We obtain the inequality

$$
\begin{array}{r}
x\left(\delta\left(V_{0}, \ldots, V_{5}\right) \backslash F\right)+\sum_{j \in V \backslash\{1\}} y_{1 j}+\sum_{j \in V \backslash\{2\}} y_{2 j}+\sum_{j \in V \backslash\{3,5\}} y_{3 j} \\
+\sum_{j \in V \backslash\{4,6\}} y_{4 j}+\sum_{j \in V \backslash\{7\}} y_{7 j} \geq 4
\end{array}
$$

which is a valid inequality. This inequality cuts off the fractional solution $(\bar{x}, \bar{y})$ since

$$
\begin{gathered}
\bar{x}\left(\delta\left(V_{0}, \ldots, V_{5}\right) \backslash F\right)+\sum_{j \in V \backslash\{1\}} \bar{y}_{1 j}+\sum_{j \in V \backslash\{2\}} \bar{y}_{2 j}+\sum_{j \in V \backslash\{3,5\}} \bar{y}_{3 j} \\
+\sum_{j \in V \backslash\{4,6\}} \bar{y}_{4 j}+\sum_{j \in V \backslash\{7\}} \bar{y}_{7 j} \\
=\bar{x}_{13}+\bar{x}_{16}+\bar{x}_{17}+\bar{x}_{25}+\bar{x}_{26}+\bar{x}_{47}=3.5<4 .
\end{gathered}
$$

In the following theorem, we define formally the family of extended $F$-partition inequalities and prove its validity.

Let $V_{0}, \ldots, V_{p}$ be a partition of $V$ such that $V_{l} \neq \varnothing$, for $l=0, \ldots, p$ and $0 \in V_{0}$. Let $i_{l} \in V_{l}$ be a fixed node for $l=1, \ldots, p$ and $F \subseteq \delta\left(V_{0}\right)$ such that $|F|=2 k+1$ for some $k \geq 0$ and integer. Let $\delta\left(V_{0}, \ldots, V_{p}\right)$ be the set of edges whose endpoints are in different sets of the partition. Consider the inequality

$$
x\left(\delta\left(V_{0}, \ldots, V_{p}\right) \backslash F\right)+\sum_{l=1}^{p} \sum_{j \in V \backslash V_{l}} y_{i_{l} j} \geq p-k .
$$

Theorem 8. Inequality (16) is valid for $\mathcal{P}$.

Proof. The following inequalities are valid for $\mathcal{P}$ :

$$
\begin{aligned}
x\left(\delta\left(V_{l}\right)\right)+2 \sum_{j \in V \backslash V_{l}} y_{i_{l} j} \geq 2 \quad l=1, \ldots, p \\
-x_{e} \geq-1 \quad \forall e \in F \\
x_{e} \geq 0 \quad \forall e \in \delta\left(V_{0}\right) \backslash F .
\end{aligned}
$$

Adding up these inequalities and dividing the resulting inequality by 2 yields

$$
x\left(\delta\left(V_{0}, \ldots, V_{p}\right) \backslash F\right)+\sum_{l=1}^{p} \sum_{j \in V \backslash V_{l}} y_{i_{l} j} \geq p-\frac{|F|}{2}
$$

As $|F|$ is odd, rounding up the right hand side yields inequality (16).

Inequalities of type (16) will be called extended $F$ partition inequalities. Note that, if values of all assignment variables are zero, i.e., if all nodes are selected as hubs, then the extended $F$-partition inequalities are the same as the $F$-partition inequalities of 2-edge connected subgraph problem.

Next, we give sufficient conditions for the extended $F$ partition inequalities to be facet defining for $\mathcal{P}$. For $\bar{A} \subseteq A$, let $X_{\bar{A}}=\left\{(x, y) \in X: y_{a}=0 \forall a \in A \backslash \bar{A}\right\}$ and $\mathcal{P}_{\bar{A}}=\operatorname{conv}\left(X_{\bar{A}}\right)$. Suppose that $\alpha x+\beta y \geq \xi$ is a facet defining inequality for $\mathcal{P}_{\bar{A}}$. Let $a \in A \backslash \bar{A}$ and $\bar{A}^{\prime}=\bar{A} \cup\{a\}$. Then the inequality

$$
\alpha x+\beta y+b_{a} y_{a} \geq \xi
$$

is facet defining for $\mathcal{P}_{\bar{A}^{\prime}}$ where $b_{a}=\xi-\theta_{a}\left(\bar{A}^{\prime}\right)$ and $\theta_{a}\left(\bar{A}^{\prime}\right)=$ $\min \left\{\alpha x+\beta y:(x, y) \in X_{\bar{A}^{\prime}}\right.$ and $\left.y_{a}=1\right\}[27]$.

Theorem 9. Inequality (16) defines a facet for $\mathcal{P}$ if the following conditions are all satisfied

(a) $G\left(V_{l}\right)$ is 3-edge connected for $l=0, \ldots, p$,

(b) $\left|F \cap \delta\left(V_{l}\right)\right| \leq 1$ and $F \cap \delta(j)=\emptyset$ for $l=1, \ldots, p$ and $j \in V_{l} \backslash\left\{i_{l}\right\}$,

(c) $|F \cap \delta(j)| \leq 1$ for $j \in V_{0} \backslash\{0\}$.

Proof. For simplicity, we use $\Delta, L$, and $I$ to denote $\delta\left(V_{0}, \ldots, V_{p}\right) \backslash F,\{1, \ldots, p\}$, and $\left\{i_{1}, \ldots, i_{p}\right\}$, respectively. Without loss of generality we assume that $\delta\left(i_{l}\right) \cap F \neq \varnothing$ for $l=1, \ldots, 2 k$. Note that from Condition $b$ ), we have $2 k+1 \leq p$.

For $\bar{A}=\emptyset, \mathcal{P}_{\bar{A}}$ reduces to the 2-edge connected subgraph polytope. Because $G\left(V_{l}\right)$ is 3-edge connected for $l=0, \ldots, p$ and $G=(V, E)$ is complete, from [25] it follows that $x(\Delta) \geq$ $p-k$ is a facet defining inequality for $\mathcal{P}_{\bar{A}}$.

If $p>2 k+1$, we let $E_{1}=\cup_{l=0}^{p} E\left(V_{l}\right) \cup\left\{i_{1}, i_{2 k+1}\right\} \cup$ $\left\{i_{2}, i_{p}\right\} \cup_{l=2 k+1}^{p-1}\left\{i_{l}, i_{l+1}\right\} \cup_{l=2}^{k}\left\{i_{2 l-1}, i_{2 l}\right\} \cup F$ and $x=\sum_{e \in E_{1}} \chi_{e}$. Clearly, $(x, 0) \in X$. If $p=2 k+1$, let $E_{2}=\cup_{l=0}^{p} E\left(V_{l}\right) \cup_{l=2}^{k}$ $\left\{i_{2 l}, i_{2 l+1}\right\} \cup\left\{i_{1}, i_{2}\right\} \cup\left\{i_{2}, i_{3}\right\} \cup F$. Then $\left(\sum_{e \in E_{2}} \chi_{e}, 0\right) \in X$. Here we give the proof for the case where $p>2 k+1$. The proof for the other case is similar.

Let $A_{1}=\{(u, v) \in A: u \in V \backslash I, v \in V\} \cup\{(u, v) \in A$ : $u=i_{l}$ for some $\left.l \in L, v \in V_{l}\right\}$. We first show that the lifting coefficients of the variables associated with the arcs of $A_{1}$ are zero. The proof is by induction. Let $(u, v) \in A_{1}$ be the first arc in the lifting sequence. Then

$$
b_{u v}=p-k-\theta_{(u, v)}(\{(u, v)\}) \text {. }
$$

Inequality (16) implies that $\theta_{(u, v)}(\{(u, v)\}) \geq p-k$. Let $x_{1}=$ $x-\sum_{e \in \delta(u)} x_{e} \chi_{e}$. Suppose that $u \notin I$. Note that if $u \in V_{0}$ and there exists $f \in F$ with $u \in f$, then we can rearrange the partition subsets so that $f \in \delta\left(V_{2 k+1}\right)$. Hence $\left(x_{1}, \gamma_{u v}\right) \in$ $X_{\{(u, v)\}}$. If $u \in I$, then without loss of generality, we may assume that $u=i_{2 k+1}$. Therefore $\left(x_{1}+\chi_{i_{1} v}+\chi_{v i_{2 k+2}}, \gamma_{u v}\right) \in$ $X_{\{(u, v)\}}$. In both cases, $\theta_{(u, v)}(\{(u, v)\})=p-k$ and thus $b_{u v}=$ 0 . In consequence $x(\Delta) \geq p-k$ is facet defining for $\mathcal{P}_{\{(u, v)\}}$. Now, let $\bar{A}_{1} \subseteq A_{1}$ be the set of arcs for which the lifting has already been done and $(u, v) \in A_{1} \backslash \bar{A}_{1}$. We assume that $b_{a}=0$ for every $a \in \bar{A}_{1}$ and show that $b_{u v}=0$. Here $b_{u v}=p-k-\theta_{(u, v)}\left(\bar{A}_{1} \cup\{(u, v)\}\right)$. Clearly, by inequality (16) 
$\theta_{(u, v)}\left(\bar{A}_{1} \cup\{(u, v)\}\right) \geq p-k$. Using the same approach as above, we can similarly show that $\theta_{(u, v)}\left(\bar{A}_{1} \cup\{(u, v)\}\right)=p-k$, and thus $b_{u v}=0$. Hence, $x(\Delta) \geq p-k$ is facet defining for $\mathcal{P}_{A_{1}}$.

Let $A_{2}=A \backslash A_{1}$. We show that the lifting coefficients of the variables associated with the arcs of $A_{2}$ are one. Let $(u, v) \in$ $A_{2}$ be the first arc in the sequence. Then

$$
b_{u v}=p-k-\theta_{(u, v)}\left(A_{1} \cup\{(u, v)\}\right) .
$$

Without loss of generality, we may assume that $u=i_{2 k+1}$. Note that $v \in V \backslash V_{2 k+1}$. First observe that by inequality (16) we have $\theta_{(u, v)}\left(A_{1} \cup\{(u, v)\}\right) \geq p-k-1$. Let $x_{2}=x-\sum_{e \in E\left(V_{2 k+1}\right)} \chi_{e}-\sum_{e \in \delta(u)} x_{e} \chi_{e}+\chi_{i_{1} i_{2 k+2}}$, and $y=\sum_{i \in V_{2 k+1} \backslash\{u\}} \gamma_{i 0}+\gamma_{u v}$. Then $\left(x_{2}, y\right) \in X_{A_{1} \cup\{(u, v)\}}$. So $\theta_{(u, v)}\left(A_{1} \cup\{(u, v)\}\right)=p-k-1$ and thus $b_{u v}=1$. Hence, $x(\Delta)+y_{u v} \geq p-k$ is facet defining for $\mathcal{P}_{A_{1} \cup\{(u, v)\}}$. Let $\bar{A}_{2} \subseteq A_{2}$ be the set of arcs for which the lifting has already been done and $(u, v) \in A_{2} \backslash \bar{A}_{2}$. We assume that $b_{a}=1$ for every $a \in \bar{A}_{2}$ and show that $b_{u v}=1$. We have

$$
b_{u v}=p-k-\theta_{(u, v)}\left(A_{1} \cup \bar{A}_{2} \cup\{(u, v)\}\right) .
$$

By inequality (16), we have $\theta_{(u, v)}\left(A_{1} \cup \bar{A}_{2} \cup\{(u, v)\}\right) \geq$ $p-k-1$. In addition, $\left(x_{2}, y\right) \in X_{A_{1} \cup \bar{A}_{2} \cup\{(u, v)\}}$. So $\theta_{(u, v)}\left(A_{1} \cup\right.$ $\left.\bar{A}_{2} \cup\{(u, v)\}\right)=p-k-1$ and $b_{u v}=1$. Therefore, $x(\Delta)+$ $\sum_{l \in L} \sum_{j \in V \backslash V_{l}} y_{i, j} \geq p-k$ is facet defining for $\mathcal{P}_{A}$.

To conclude this section, we prove that the separation problem associated with the extended $F$-partition inequalities (16) is NP-hard. Here we consider the problem of finding a most violated inequality. So we define the decision version of the separation problem as follows. Given a graph $G=(V, E)$, a special node $0 \in V$, a solution $(\bar{x}, \bar{y}) \in R_{+}^{|E|} \times R_{+}^{|V|^{2}}$, and a positive number $\kappa$, does there exist a partition $V_{0}, V_{1}, \ldots, V_{p}$ of $V$ with $0 \in V_{0}, F \subseteq \delta\left(V_{0}\right)$ with $|F|=2 k+1$ for some integer $k \geq 0, i_{l} \in V_{l}$ for $l=1, \ldots, p$ such that $\bar{x}\left(\delta\left(V_{0}, \ldots, V_{p}\right) \backslash \bar{F}\right)+\sum_{l=1}^{p} \sum_{j \in V \backslash V_{l}} \bar{y}_{i j} \leq p-k-\kappa$ ?

To establish the complexity status of the separation problem associated with the extended $F$-partition inequalities, we will use a reduction from the decision version of the uncapacitated concentrator location problem (decUCL). The decUCL is defined as follows. Given a set of nodes $I$, cost $C_{i j}$ for $i \in I$ and $j \in I$ and a positive scalar $K$, does there exist a nonempty subset $I^{\prime}$ of $I$ and a choice $j_{i} \in I^{\prime}$ for each $i \in I \backslash I^{\prime}$ such that $\sum_{j \in I^{\prime}} C_{j j}+\sum_{i \in I \backslash I^{\prime}} C_{i j_{i}} \leq K$ ? This problem is NP-complete [22]. To avoid trivial cases, we consider instances with $\max _{i, j \in I} C_{i j}>\frac{K}{|I|}$.

Theorem 10. The decision version of the separation problem associated with the extended $F$-partition inequalities (16) is NP-complete.

Proof. It is easy to verify that the problem is in NP.

To show that the problem is NP-complete, we give a polynomial time reduction of the decUCL to the decision version of the separation problem. Given an instance of the
decUCL, consider the following instance of the separation problem. Let $\kappa=\max _{i, j \in I} C_{i j}|I|-K$. Set $V=\{0\} \cup I$, $E=\{\{i, j\}: i \in V, j \in V \backslash\{i\}\}, \bar{x}=0, \bar{y}_{i j}=\frac{K+\kappa}{|I|}-C_{j i}$ for $i \in I$ and $j \in I$. Observe that $\bar{y}_{i j}$ is non-negative for each $i \in I$ and $j \in I$. Let $\bar{y}_{i 0}=\bar{y}_{0 i}=0$ for all $i \in I$ and $\bar{y}_{00}=1$.

Notice that as $\bar{x}=0$ and $\sum_{j \in V \backslash V_{l}} y_{i_{l} j}=1-\sum_{j \in V_{l}} y_{i_{j} j}$ for $i_{l}$ for $l=1, \ldots, p$, the extended $F$-partition inequality (16) can be rewritten as

$$
\sum_{l=1}^{p} \sum_{j \in V_{l}} y_{i_{l} j} \leq k .
$$

Let $V_{0}, V_{1}, \ldots, V_{p}$ be a partition of $V$ with $0 \in V_{0}, F \subseteq$ $\delta\left(V_{0}\right)$ with $|F|=2 k+1$ for some integer $k \geq 0, i_{l} \in V_{l}$ for $l=1, \ldots, p$ such that the corresponding extended $F$ partition inequality (17) is violated with violation at least as large as $\kappa$. Now consider a new partition such that $V_{l}^{\prime}=V_{l}$ for $l=2, \ldots, p, V_{0}^{\prime}=\{0\}$, and $V_{1}^{\prime}=V_{1} \cup V_{0} \backslash\{0\}$. Let $F^{\prime}$ be a subset of $\delta(0)$ with cardinality 1 . The resulting extended $F$-partition inequality (17) is also violated with violation at least as large as $\kappa$. Hence, there exists an extended $F$-partition inequality with violation at least $\kappa$ if and only if there exists an extended $F$-partition inequality with violation at least $\kappa$, $V_{0}=\{0\}$, and $F \subseteq \delta(0)$ with $|F|=1$.

We claim that there exists a solution to the decUCL if and only if there exists a partition of $V$ into $V_{0}, V_{1}, \ldots, V_{p}$ with $V_{0}=\{0\}$ and $F \subseteq \delta(0)$ with $|F|=1$, and a choice of nodes $i_{1}, \ldots, i_{p}$ with $i_{l} \in V_{l}$ for $l=1, \ldots, p$ such that the inequality (17) is violated with a violation of at least $\kappa$.

Given a solution of decUCL, let $p=\left|I^{\prime}\right|$ and so $I^{\prime}=$ $\left\{i_{1}, \ldots, i_{p}\right\}$. For $l=1, \ldots, p$, let $V_{l}=\left\{i \in I \backslash I^{\prime}: j_{i}=\right.$ $\left.i_{l}\right\} \cup\left\{i_{l}\right\}, V_{0}=\{0\}$ and $F$ be any element of $\delta(0)$. The left- hand side of the inequality, $\sum_{l=1}^{p} \sum_{j \in V_{l}} \bar{y}_{i j}$, is equal to $\sum_{l=1}^{p} \bar{y}_{i i_{l}}+$ $\sum_{l=1}^{p} \sum_{j \in V_{l} \backslash\left\{i_{i}\right\}} \bar{y}_{i, j}=\sum_{i \in I^{\prime}}\left(\frac{K+\kappa}{|I|}-C_{i i}\right)+\sum_{i \in I \backslash I^{\prime}}\left(\frac{K+\kappa}{|I|}+\right.$ $\left.C_{i j_{i}}\right)=K+\kappa-\left(\sum_{i \in I^{\prime}} C_{i i}+\sum_{i \in I \backslash I^{\prime}} C_{i j_{i}}\right)$. As $\sum_{i \in I^{\prime}} C_{i i}+$ $\sum_{i \in I \backslash I^{\prime}} C_{i j_{i}} \leq K$, we have $\sum_{l=1}^{p} \sum_{j \in V_{l}} \bar{y}_{i j} \geq \kappa$ and hence the inequality (17) is violated with a violation of at least $\kappa$.

Given a partition of $V$ into $V_{0}, V_{1}, \ldots, V_{p}$ with $V_{0}=\{0\}$, $F \subseteq \delta(0)$ with $|F|=1$, and a choice of nodes $i_{1}, \ldots, i_{p}$ with $i_{l} \in V_{l}$ for $l=1, \ldots, p$ such that the inequality (17) is violated with a violation of at least $\kappa$, let $I^{\prime}=\left\{i_{1}, \ldots, i_{p}\right\}$, and $j_{k}=i_{l}$ for $k \in V_{l}$ and $l \in\{1, \ldots, p\}$. We can show that this is a solution to the decUCL following the steps above.

In Section 5, we propose several heuristic algorithms for the separation of the extended $F$-partition (16) inequalities.

\subsection{Star-Path Inequalities}

In this section, we introduce a new family of facet defining inequalities called the star-path inequalities. These generalize constraints (8). We first give an example of a fractional solution and a star-path inequality that cuts off this solution.

Let $V=\{0, \ldots, 5\}$. Consider the fractional point $(\bar{x}, \bar{y})$ depicted in Figure 3. Here we omit the edges and arcs with value equal to 0 . The remaining edges and arcs have values 


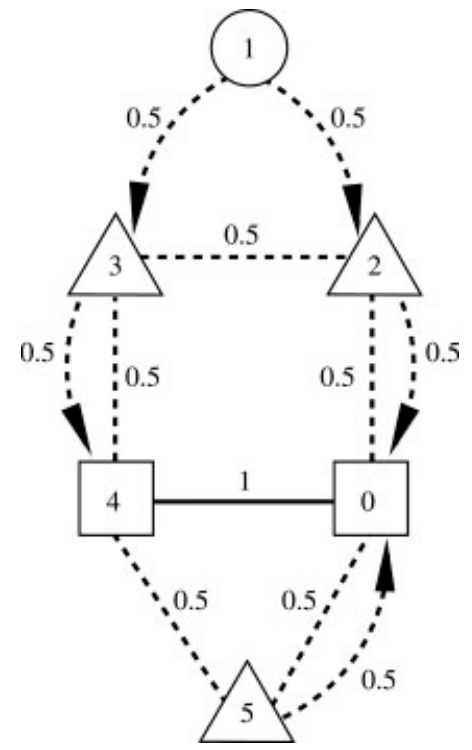

FIG. 3. The backbone edges and assignment arcs in the fractional solution $(\bar{x}, \bar{y})$.

0.5 or 1 . The nodes $i$ for which $\bar{y}_{i i}=1$ are represented by rectangles, those with $\bar{y}_{i i}=0.5$ are represented by triangles, and finally the nodes with $\bar{y}_{i i}=0$ are represented by ellipses. The backbone edges with value 1 are represented by bold lines and those with value 0.5 are represented by dashed lines. We use dashed lines with arrows for the assignment arcs.

Consider nodes $1-3$. Notice that for $(x, y) \in X$, if no concentrators are installed at nodes 2 and 3, i.e., $\sum_{j \in V \backslash\{2\}} y_{2 j}=1$ and $\sum_{j \in V \backslash\{3\}} y_{3 j}=1$, then the edges $\{1,2\}$ and $\{2,3\}$ cannot be used in the backbone network and node 1 cannot be assigned to either of nodes 2 or 3 , hence we must have $x_{12}+x_{23}+y_{12}+y_{13}=0$. If a concentrator is installed at node 3 but not at node 2, i.e., $\sum_{j \in V \backslash\{2\}} y_{2 j}=1$ and $\sum_{j \in V \backslash\{3\}} y_{3 j}=0$, then as the edges $\{1,2\}$ and $\{2,3\}$ cannot be in the backbone and node 1 cannot be assigned to node 2, we have $x_{12}+x_{23}+y_{12}=0$ and $y_{13}$ can be 0 or 1 . If the opposite happens, i.e., $\sum_{j \in V \backslash\{2\}} y_{2 j}=0$ and $\sum_{j \in V \backslash\{3\}} y_{3 j}=1$, then $x_{23}+y_{13}=0$ and $x_{12}$ and $y_{12}$ can be 0 or 1 , but we must have $x_{12}+y_{12} \leq 1$. Finally, if concentrators are installed at both nodes 2 and 3, i.e., $\sum_{j \in V \backslash\{2\}} y_{2 j}=0$ and $\sum_{j \in V \backslash\{3\}} y_{3 j}=0$, then $x_{12}+y_{12}+y_{13} \leq 1$ and $x_{23}$ can be 0 or 1 . Hence, the inequality

$$
x_{12}+x_{23}+y_{12}+y_{13}+\sum_{j \in V \backslash\{2\}} y_{2 j}+\sum_{j \in V \backslash\{3\}} y_{3 j} \leq 2
$$

is valid for $\mathcal{P}$.

Now notice that $\bar{x}_{12}+\bar{x}_{23}+\bar{y}_{12}+\bar{y}_{13}+\sum_{j \in V \backslash\{2\}} \bar{y}_{2 j}+$ $\sum_{j \in V \backslash\{3\}} \bar{y}_{3 j}=2.5>2$. Hence, adding this inequality to the formulation cuts off the fractional solution $(\bar{x}, \bar{y})$.

We remark here that $x_{23}, y_{24}, y_{12}, y_{13}, y_{34}$ form an odd hole of size 5 in the conflict graph associated with $X_{S}$. Hence, the odd hole inequality $x_{23}+y_{24}+y_{12}+y_{13}+y_{34} \leq 2$ is valid for $X$ and this odd hole inequality is violated by $(\bar{x}, \bar{y})$. Inequality (18) can be obtained by lifting this odd hole inequality sequentially with $y_{2 j}$ for $j \in V \backslash\{2,4\}, y_{3 j}$ for $j \in$ $V \backslash\{3,4\}$ and $x_{12}$.

Next, we show that inequality (18) is a special case of a more general family of valid inequalities. Let $m \geq 1$ be an integer and $I_{m}=\left\{i_{0}, \ldots, i_{m}\right\}$ be an ordered subset of $V \backslash\{0\}$ consisting of distinct nodes. Let $P_{I}=\left\{\left\{i_{l}, i_{l+1}\right\} \in E\right.$ : $i=0, \ldots, m-1\}$. Note that $P_{I}$ is a path between $i_{0}$ and $i_{m}$. Consider the inequality

$$
x\left(P_{I}\right)+\sum_{i \in I \backslash\left\{i_{0}\right\}} \sum_{(i, j) \in A} y_{i j}+\sum_{j \in I:\left(i_{0}, j\right) \in A} y_{i_{0} j} \leq m
$$

Theorem 11. Inequality (19) is valid for $\mathcal{P}$.

Proof. We will prove the validity by induction on $m=$ $\left|P_{I_{m}}\right|$. If $m=1$, the star-path inequality reduces to

$$
x_{i_{0} i_{1}}+\sum_{j \in V \backslash\left\{i_{1}\right\}} y_{i_{1} j}+y_{i_{0} i_{1}} \leq 1
$$

which is nothing but constraint (8) for $\left(i_{0}, i_{1}\right)$ and hence it is valid for $\mathcal{P}$.

Now assume that the star-path inequalities are valid for $m \leq k$. By the induction hypothesis,

$$
x\left(P_{I_{k}}\right)+\sum_{l=1}^{k} \sum_{j \in V \backslash\left\{i_{l}\right\}} y_{i_{l} j}+\sum_{l=1}^{k} y_{i_{0} i_{l}} \leq k
$$

holds for any $(x, y) \in \mathcal{P}$. If $x_{i_{k} i_{k+1}}+\sum_{j \in V \backslash\left\{i_{k+1}\right\}} y_{i_{k+1} j}+$ $y_{i_{0} i_{k+1}} \leq 1$, then summing this with the above inequality gives $x\left(P_{I_{k+1}}\right)+\sum_{l=1}^{k+1} \sum_{j \in V \backslash\left\{i_{l}\right\}} y_{i j j}+\sum_{l=1}^{k+1} y_{i_{0} i_{l}} \leq k+1$.

If $x_{i_{k}, i_{k+1}}+\sum_{j \in V \backslash\left\{i_{k+1}\right\}} y_{i_{k+1} j}+y_{i_{0} i_{k+1}} \geq 2$, then, as we know that $x_{i_{k}, i_{k+1}}+\sum_{j \in V \backslash\left\{i_{k+1}\right\}} y_{i_{k+1} j} \leq 1$ and $\sum_{j \in V \backslash\left\{i_{k+1}\right\}} y_{i_{k+1} j}+$ $y_{i_{0} i_{k+1}} \leq 1, x_{i_{k}, i_{k+1}}=1, \sum_{j \in V \backslash\left\{i_{k+1}\right\}} y_{i_{k+1} j}=0$, and $y_{i_{0} i_{k+1}}=1$. This implies that $x_{i_{0} i_{1}}=0$ and $\sum_{l=1}^{k+1} y_{i_{0} i_{l}}=1$. Moreover, we have that the inequalities $x_{i, i_{l+1}}+\sum_{j \in V \backslash\left\{i_{l}\right\}} y_{i, j} \leq 1$ are valid for $l=1, \ldots, k$. Summing up these inequalities together with $x_{i_{0} i_{1}}=0, \sum_{l=1}^{k+1} y_{i_{0} i_{l}}=1$ and $\sum_{j \in V \backslash\left\{i_{k+1}\right\}} y_{i_{k+1} j}=0$ yields $x\left(P_{I_{k+1}}\right)+\sum_{l=1}^{k+1} \sum_{j \in V \backslash\left\{i_{i}\right\}} y_{i j}+\sum_{l=1}^{k+1} y_{i_{0} i_{l}} \leq k+1$. Hence, inequality (19) is valid for $\mathcal{P}$.

Inequalities of type (19) will be called star-path inequalities. Observe that inequalities (8) represent a special case of star-path inequalities. Moreover, by Corollary 1, the former ones are facet defining for $\mathcal{P}$.

Theorem 12. If $|V \backslash I| \geq 3$, then inequality (19) is facet defining for $\mathcal{P}$.

Proof. Let $\mathcal{F}=\left\{(x, y) \in \mathcal{P}: x\left(P_{I}\right)+\right.$ $\left.\sum_{l=1}^{m} \sum_{j \in V \backslash\left\{i_{i}\right\}} y_{i_{l} j}+\sum_{l=1}^{m} y_{i_{0} i_{l}}=m\right\}$. Assume that every solution $(x, y) \in \mathcal{F}$ also satisfies $a x+b y=\beta$. For $l=0, \ldots, m$, define $V_{0 l}=\left\{i_{0}, \ldots, i_{l}\right\}, V_{l m}=\left\{i_{l}, \ldots, i_{m}\right\}$, $x^{l}=\sum_{e \in E\left(V \backslash V_{l m}\right)} \chi_{e}, \bar{x}^{l}=\sum_{e \in E\left(V \backslash V_{0 l}\right)} \chi_{e}$.

Let $x=\sum_{e \in E} \chi_{e}$. The solution $(x, 0)$ is in $\mathcal{F}$. Let $e \in$ $E \backslash P_{I}$. As the solution $\left(x-\chi_{e}, 0\right)$ is also in $\mathcal{F}$, we have $a_{e}=0$. 
Let $j \in V \backslash V_{0 m}$ and $k \in V \backslash\{j\}$. The solution $(x-$ $\left.\sum_{e \in \delta(j)} \chi_{e}, \gamma_{j k}\right)$ is also in $\mathcal{F}$. Hence $b_{j k}=0$.

Let $k \in V \backslash\left\{i_{m}\right\}$. As both solutions $(x, 0)$ and $\left(x^{m}, \gamma_{i_{m} k}\right)$ are in $\mathcal{F}$ and $a_{e}=0$ for all $e \in E \backslash P_{I}$, we have $a_{i_{m-1} i_{m}}=b_{i_{m} k}=\sigma_{m}$ for all $k \in V \backslash\left\{i_{m}\right\}$ for some $\sigma_{m} \in \mathbb{R}$.

Let $l \in\{1, \ldots, m-1\}$ and $k \in V \backslash V_{l m}$. As both solutions $\left(x^{l+1}, \sum_{j=l+1}^{m} \gamma_{i_{j} 0}\right)$ and $\left(x^{l}, \sum_{j=l+1}^{m} \gamma_{i_{j} 0}+\gamma_{i_{l} k}\right)$ are in $\mathcal{F}$, we can conclude that $a_{i_{l-1}, i_{l}}=b_{i_{l} k}=\sigma_{l}$ for all $k \in V \backslash V_{l m}$ for some $\sigma_{m} \in R$.

Let $k \in V \backslash V_{0 m}$. As both solutions $\left(x^{1}, \sum_{j=1}^{m} \gamma_{i_{j} 0}\right)$ and $\left(x^{0}, \sum_{j=1}^{m} \gamma_{i_{j} 0}+\gamma_{i_{0} k}\right)$ are both in $\mathcal{F}$, we can conclude that $b_{i_{0} k}=0$ for all $k \in V \backslash V_{0 m}$.

Let $k \in V_{1 m}$. Consider the solutions $(x, 0)$ and $\left(\bar{x}^{0}, \gamma_{i_{0}, k}\right)$. As both of these solutions are in $\mathcal{F}$, we have $b_{i_{0} k}=a_{i_{0}, i_{1}}=\sigma_{1}$.

Let $l \in\{1, \ldots, m-1\}$ and $k \in V_{l+1, m}$. Solutions $\left(\bar{x}^{l-1}, \sum_{j \in V_{0 l-1}} \gamma_{i_{j}, i_{m}}\right)$ and $\left(\bar{x}^{l}, \sum_{j \in V_{0 l-1}} \gamma_{i_{j}, i_{m}}+\gamma_{i_{l} k}\right)$ are both in $\mathcal{F}$. Hence $a_{i_{l}, i_{l}+1}=b_{i_{l}, k}=\sigma_{l}$ for all $k \in V_{l+1, m}$.

Now as $a_{i_{l-1}, i_{l}}=\sigma_{l}=a_{i_{l}, i_{l}+1}$ for all $l \in\{1, \ldots, m-1\}$, we have $\sigma_{l}=\sigma$ for all $l \in\{1, \ldots, m\}$.

This proves that $a x+b y=\beta$ is a multiple of $x\left(P_{I}\right)+$ $\sum_{l=1}^{m} \sum_{j \in V \backslash\left\{i_{l}\right\}} y_{i_{l} j}+\sum_{l=1}^{m} y_{i_{0} i_{l}}=m$.

Next, we prove that the separation problem associated with the star-path inequalities (19) is NP-hard. First, we remark that inequality (19) can be rewritten as $x\left(P_{I}\right)-\sum_{l=1}^{m} y_{i_{l} i_{l}}+$ $\sum_{l=1}^{m} y_{i_{0} i_{l}} \leq 0$ using the self assignment variables.

The decision version of the separation problem is then defined as follows. Given a graph $G=(V, E), K>0$, a special node $0 \in V$, a solution $(\bar{x}, \bar{y}) \in R_{+}^{|E|} \times R_{+}^{|V|^{2}}$, does there exist a set of $m+1$ distinct nodes $i_{0}, i_{1}, \ldots, i_{m}$ in $V \backslash\{0\}$ such that $\bar{x}\left(P_{I}\right)-\sum_{l=1}^{m} \bar{y}_{i_{l} i_{l}}+\sum_{l=1}^{m} \bar{y}_{i_{0} i_{l}} \geq K$ ?

Theorem 13. The decision version of the separation problem associated with the star-path inequalities (19) is NP-complete.

Proof. NP membership is easily verifiable. To establish the complexity status we give a reduction from the Hamiltonian path problem, which is defined as follows. Given a graph $G^{\prime}=\left(V^{\prime}, E^{\prime}\right)$, does $G^{\prime}$ contain a simple path consisting of all the nodes in $V^{\prime}$ ? Given such an instance, consider the following instance of the separation problem. Set $V=V^{\prime} \cup\{0\}$, $E=\{\{i, j\}: i \in V, j \in V \backslash\{i\}\} \bar{x}_{e}=1$ for $e \in E^{\prime}, \bar{x}_{e}=0$ for $e \in E \backslash E^{\prime}, \bar{y}=0$, and $K=|V|-1$. Now it is easy to conclude that $G^{\prime}$ has a Hamiltonian path if and only if the constructed separation problem has a solution.

\section{REDUCTION OPERATIONS}

In this section, we are going to introduce some reduction operations, which will be used in our branch-and-cut algorithm that will be discussed in the next section. These operations use ideas developed by Fonlupt and Mahjoub [7] for the 2-edge connected subgraph polytope.

Note that both an undirected graph $G=(V, E)$ and a directed graph $D=(V, A)$ are associated with the problem 2ECSSP. The reduction operations may affect both. Given $e=u v \in E$, contracting $e$ means deleting $e$ from $E$ and arcs $(u, v),(v, u)$ from $A$, identifying $u$ and $v$, deleting the resulting loops, and keeping the new parallel edges and arcs. Similarly contracting a set of nodes $W \subset V$ means deleting set of edges $E(W)$ and set of $\operatorname{arcs} A(W)$, identifying $W$ as a single node, deleting the resulting loops and keeping the new parallel edges and arcs.

Before describing these operations, we shall first introduce some notation. We will denote by $Q(G)$ the polytope given by inequalities (8)-(12). That is to say, $Q(G)$ is the linear relaxation of $2 \operatorname{ECSSP}(G)$. Clearly, $Q(G)$ is defined in terms of both graphs $G$ and $D$. However, as $G$ and $D$ are closely related, we will only write $Q(G)$ for $Q(G, D)$. If $(\bar{x}, \bar{y})$ is a solution of $Q(G)$ we will denote by $E_{0}(\bar{x}), E_{1}(\bar{x})$, and $E_{f}(\bar{x})$, the set of edges $e \in E$ such that $\bar{x}(e)=0, \bar{x}(e)=1$, and $0<\bar{x}(e)<1$, respectively. Similarly, we will denote by $A_{0}(\bar{y}), A_{1}(\bar{y})$, and $A_{f}(\bar{y})$ the set of arcs $a \in A$ with $\bar{y}(a)=$ $0, \bar{y}(a)=1$, and $0<\bar{y}(a)<1$, respectively. We also use $\Gamma(\bar{x}, \bar{y}), T(\bar{x}, \bar{y}), \xi(\bar{x}, \bar{y})$ to denote the set of $\operatorname{arcs}$ of $A$, nodes of $V \backslash\{0\}$, and pairs $(S, i)$ for all $S \subseteq V \backslash\{0\}, i \in S$, respectively, for which the corresponding inequalities (8)-(10) are tight for $(\bar{x}, \bar{y})$.

Let $(\bar{x}, \bar{y})$ be an extreme point of $Q(G)$. Thus there is a set of $\operatorname{arcs} \Gamma^{*}(\bar{x}, \bar{y}) \subseteq \Gamma(\bar{x}, \bar{y})$, a set of nodes $T^{*}(\bar{x}, \bar{y}) \subseteq T(\bar{x}, \bar{y})$ and a set $\xi^{*}(\bar{x}, \bar{y}) \subseteq \xi(\bar{x}, \bar{y})$ such that $(\bar{x}, \bar{y})$ is the unique solution of the system

$$
R(\bar{x}, \bar{y})= \begin{cases}x_{i j}+y_{i j}+\sum_{k \in V \backslash\{j\}} y_{j k}=1 & (i, j) \in \Gamma^{*}(\bar{x}, \bar{y}), \\ x_{0 i}+\sum_{k \in V \backslash\{i\}} y_{i k}=1 & i \in T^{*}(\bar{x}, \bar{y}), \\ x(\delta(S))+2 \sum_{j \in V \backslash S} y_{i j}=2 & (S, i) \in \xi^{*}(\bar{x}, \bar{y}), \\ x_{i j}=0 & i j \in E_{0}(\bar{x}), \\ x_{i j}=1 & i j \in E_{1}(\bar{x}), \\ y_{i j}=0 & (i, j) \in A_{0}(\bar{y}), \\ y_{i j}=1 & (i, j) \in A_{1}(\bar{y}) .\end{cases}
$$

Note that the nontrivial equations of $R(\bar{x}, \bar{y})$ must have at least two variables with fractional values (note that the right hand side of each of these equations is integer). If all the variables of one of these equations have value 0 or 1 , then that inequality would be redundant with respect to $x_{i j}=0$, $i j \in E_{0}(\bar{x}), x_{i j}=1, i j \in E_{1}(\bar{x}), y_{i j}=0,(i, j) \in A_{0}(\bar{y})$ and $y_{i j}=1,(i, j) \in A_{1}(\bar{y})$.

Let $(\bar{x}, \bar{y})$ be a solution of $Q(G)$. Consider the following operations with respect to $(\bar{x}, \bar{y})$ :

- $\theta_{1}$ : Delete an edge $e$ with $\bar{x}_{e}=0$.

- $\theta_{2}$ : Delete an arc $(i, j)$ with $\bar{y}_{i j}=0$.

- $\theta_{3}$ : Delete a node $i$ as well as all the edges and arcs incident to it, if there is some $j$ such that $\bar{y}_{i j}=1$.

- $\theta_{4}$ : Contract a node set $W$ such that $G(W)$ is 2-edge connected and $\bar{x}_{e}=1$ for every $e \in E(W)$.

- $\theta_{5}$ : Contract an edge $e$ if at least one of the endpoints of $e$ is incident to exactly two edges, and these two edges have value 1 with respect to $\bar{x}$.

Note that the edges and the arcs with fractional values are preserved by all the reduction operations. Note also that $\theta_{1}$ 
and $\theta_{2}$ modify only $G$, whereas the remaining ones affect both $G$ and $D$. Starting from $G=(V, E)$ and $D=(V, A)$ and applying repeatedly $\theta_{1}, \ldots, \theta_{5}$, we obtain reduced graphs $G^{\prime}=\left(V^{\prime}, E^{\prime}\right), D^{\prime}=\left(V^{\prime}, A^{\prime}\right)$ and a solution $\left(\bar{x}^{\prime}, \bar{y}^{\prime}\right) \in Q\left(G^{\prime}\right)$. We remark that $\left(\bar{x}^{\prime}, \bar{y}^{\prime}\right)$ is nothing but the restriction of $(\bar{x}, \bar{y})$ in $G^{\prime}$ and $D^{\prime}$. We have the following:

Theorem 14. $(\bar{x}, \bar{y})$ is an extreme point of $\mathcal{Q}(G)$ if and only if $\left(\bar{x}^{\prime}, \bar{y}^{\prime}\right)$ is an extreme point of $\mathcal{Q}\left(G^{\prime}\right)$.

Proof. Suppose $(\bar{x}, \bar{y})$ is an extreme point of $Q(G)$. Without loss of generality, we may suppose that $\left(\bar{x}^{\prime}, \bar{y}^{\prime}\right)$ is obtained by the application of $\theta_{1}, \ldots, \theta_{5}$ exactly once. It is clear that if $\left(\bar{x}^{\prime}, \bar{y}^{\prime}\right)$ is obtained by either operation $\theta_{1}$ or $\theta_{2}$, then $\left(\bar{x}^{\prime}, \bar{y}^{\prime}\right)$ is an extreme point of $Q\left(G^{\prime}\right)$. Now suppose that $\bar{y}_{i j}=1$ and that $\left(\bar{x}^{\prime}, \bar{y}^{\prime}\right)$ is obtained by the application of $\theta_{3}$ with respect to node $i$. First observe that, by inequalities (8), we have $\bar{x}_{i l}=0$ for all $\{i, l\} \in E, \bar{y}_{i l}=0$ for all $(i, l) \in A$ with $l \neq i$, and $\bar{y}_{l i}=0$ for all $(l, i) \in A$ with $l \neq i$. Moreover, it is clear that inequalities (8) and (9) with respect to $G^{\prime}$ are satisfied by $\left(\bar{x}^{\prime}, \bar{y}^{\prime}\right)$. Now consider a cut $\delta\left(S^{\prime}\right)$ of $G^{\prime}$ and a node $k \in S^{\prime}$. Note that $k \neq i$. As $(\bar{x}, \bar{y}) \in Q(G)$ we have $2 \leq \bar{x}\left(\delta_{G}\left(S^{\prime}\right)\right)+2 \sum_{l \in V \backslash S^{\prime}} \bar{y}_{k l}=\bar{x}\left(\delta_{G^{\prime}}\left(S^{\prime}\right)\right)+\sum_{l \in S^{\prime}} \bar{x}_{i l}+$ $2 \sum_{l \in V^{\prime} \backslash S^{\prime}} \bar{y}_{k l}+2 \bar{y}_{k i}=\bar{x}^{\prime}\left(\delta_{G^{\prime}}\left(S^{\prime}\right)\right)+2 \sum_{l \in V^{\prime} \backslash S^{\prime}} \bar{y}_{k l}^{\prime}$, and hence the cut inequality (10) induced by $\left(S^{\prime}, k\right)$ in $G^{\prime}$ is satisfied by $\left(\bar{x}^{\prime}, \bar{y}^{\prime}\right)$. Thus $\left(\bar{x}^{\prime}, \bar{y}^{\prime}\right)$ is a solution of $Q\left(G^{\prime}\right)$. Moreover, all the edges and arcs removed from the graph have integer values. Hence, they appear as trivial equations in system $R(\bar{x}, \bar{y})$. Consequently, $\left(\bar{x}^{\prime}, \bar{y}^{\prime}\right)$ is the unique solution of a subsystem of $R(\bar{x}, \bar{y})$, and therefore it is an extreme point of $Q\left(G^{\prime}\right)$.

Now suppose $\left(\bar{x}^{\prime}, \bar{y}^{\prime}\right)$ comes from the application of $\theta_{4}$ with respect to a node set $W$. First note that all the arcs with both endnodes in $W$ have value zero with respect to $\bar{y}$. It is easy to see that inequalities (8) and (9) remain satisfied by $\left(\bar{x}^{\prime}, \bar{y}^{\prime}\right)$ in $G^{\prime}$. Let $U^{\prime} \subseteq V^{\prime}$ and $k \in U^{\prime}$. Let $w$ be the node of $V^{\prime}$ which arises from the contraction of $W$ and, without loss of generality, suppose that $w \in U^{\prime}$. Let $U=\left(U^{\prime} \backslash\{w\}\right) \cup$ $W$. As $k \in U$ and $(\bar{x}, \bar{y})$ is a solution of $Q(G)$, we have $2 \leq \bar{x}\left(\delta_{G}(U)\right)+2 \sum_{l \in V \backslash U} \bar{y}_{k l}=\bar{x}\left(\delta_{G^{\prime}}\left(U^{\prime}\right)\right)+2 \sum_{l \in V^{\prime} \backslash U^{\prime}} \bar{y}_{k l}$, and hence the cut inequality (10) induced by $\left(U^{\prime}, k\right)$ in $G^{\prime}$ is satisfied by $\left(\bar{x}^{\prime}, \bar{y}^{\prime}\right)$. Therefore $\left(\bar{x}^{\prime}, \bar{y}^{\prime}\right)$ is a solution of $Q\left(G^{\prime}\right)$.

Now suppose, on the contrary, that $\left(\bar{x}^{\prime}, \bar{y}^{\prime}\right)$ is not an extreme point of $Q\left(G^{\prime}\right)$. Thus there exist two solutions $\left(\bar{x}^{1^{\prime}}, \bar{y}^{1^{\prime}}\right)$ and $\left(\bar{x}^{\prime}, \bar{y}^{2^{\prime}}\right)$ of $Q\left(G^{\prime}\right)$ such that $\left(\bar{x}^{\prime}, \bar{y}^{\prime}\right)=\frac{1}{2}\left(\left(\bar{x}^{1^{\prime}}, \bar{y}^{1^{\prime}}\right)+\left(\bar{x}^{2^{\prime}}, \bar{y}^{2^{\prime}}\right)\right)$. Consider the solution given by

$$
\bar{x}_{e}^{i}= \begin{cases}\bar{x}_{e}^{i^{\prime}}, & \text { for all } e \in E \backslash E(W) \\ 1, & \text { for all } e \in E(W)\end{cases}
$$

and

$$
\bar{y}_{a}^{i}= \begin{cases}\bar{y}_{a}^{i^{\prime}}, & \text { for all } a \in A^{\prime} \\ 0, & \text { otherwise }\end{cases}
$$

for $i=1,2$. Clearly, $\left(\bar{x}^{i}, \bar{y}^{i}\right) \in Q(G)$ for $i=1,2$. Moreover, $(\bar{x}, \bar{y})=\frac{1}{2}\left(\left(\bar{x}^{1}, \bar{y}^{1}\right)+\left(\bar{x}^{2}, \bar{y}^{2}\right)\right)$. This contradicts the extremality of $(\bar{x}, \bar{y})$. The proof is similar for $\theta_{5}$. Repeating a similar line of arguments, one can easily show the converse.
Theorem 14 is important from an algorithmic point of view. It shows the correspondence between the extreme points of $Q(G)$ and those of $Q\left(G^{\prime}\right)$. Thus, any algorithm for separating fractional extreme points of $Q\left(G^{\prime}\right)$ may also be used for separating the corresponding fractional extreme points of $Q(G)$.

In what follows, we shall give algorithmic consequences of the reduction operations.

Theorem 15. There is a cut inequality $(10)$ violated by $(\bar{x}, \bar{y})$ in $G$ if and only if there is a cut inequality violated by $\left(\bar{x}^{\prime}, \bar{y}^{\prime}\right)$ in $G^{\prime}$.

Proof. Let $S \subseteq V, i \in S$ and suppose that the cut inequality induced by $(S, i)$ is violated by $(\bar{x}, \bar{y})$, that is to say $\bar{x}(\delta(S))+2 \sum_{j \in V \backslash S} \bar{y}_{i j}<2$. We will show that there is a node set $S^{\prime} \subseteq V^{\prime}$ and a node $i^{\prime} \in S^{\prime}$ whose corresponding cut inequality in $G^{\prime}$ is violated by $\left(\bar{x}^{\prime}, \bar{y}^{\prime}\right)$. First, it is clear that if $\left(\bar{x}^{\prime}, \bar{y}^{\prime}\right)$ is obtained by operation $\theta_{1}$ (resp. $\theta_{2}$ ) with respect to an edge $i j$ (resp. arc $(i, j))$ such that $\bar{x}_{i j}=0\left(\right.$ resp. $\left.\bar{y}_{i j}=0\right)$, then the same inequality is violated by $\left(\bar{x}^{\prime}, \bar{y}^{\prime}\right)$ in $G^{\prime}$. Suppose $\left(\bar{x}^{\prime}, \bar{y}^{\prime}\right)$ is obtained by $\theta_{3}$ with respect to an arc $(u, w)$ with $\bar{y}_{u w}=1$. By inequality (8), we have $\bar{x}_{u v}=0$ for all $v \in V \backslash\{u\}, \bar{y}_{u v}=0$ for all $v \in V \backslash\{u, w\}$ and $\bar{y}_{w v}=0$ for all $v \in V \backslash\{w\}$. If $u \neq i$, then $(S \backslash\{u\}, i)(\operatorname{resp} .(S, i))$ induces a violated cut inequality with respect to $(\bar{x}, \bar{y})$, if $u \in S$ (resp $u \notin S$ ). That is to say we can take $S^{\prime}=S$ and $i^{\prime}=i$ if $u \notin S$, and $S^{\prime}=S \backslash\{u\}$ and $i^{\prime}=i$ if $u \in S$. If $u=i$, as $\bar{y}_{u w}=1$ and the cut inequality induced by $(S, u)$ is violated, it follows that $w \in S$. By considering $S^{\prime}=S \backslash\{u\}$ and $i^{\prime}=w$, we have that the cut inequality in $G^{\prime}$ induced by $\left(S^{\prime}, i^{\prime}\right)$ is violated.

Suppose $\left(\bar{x}^{\prime}, \bar{y}^{\prime}\right)$ is obtained by $\theta_{4}$ with respect to a node set $W \subset V$. Let $w$ be the node that arises from the contraction of $W$. Because $(W, E(W))$ is 2-edge connected and $\bar{x}_{e}=1$ for all $e \in E(W)$, we should have either $W \subseteq S$ or $W \subseteq V \backslash S$, for otherwise, the cut inequality induced by $(S, i)$ would not be violated.

If $W \subseteq S$, then set $S^{\prime}=(S \backslash W) \cup\{w\}$ and $i^{\prime}=w$ (resp. $i^{\prime}=i$ ), if $i \in W$ (resp. $i \in S \backslash W$ ).

If $W \subseteq V \backslash S$, then set $S^{\prime}=S$ and $i^{\prime}=i$.

In both cases, $\left(S^{\prime}, i^{\prime}\right)$ induces a cut inequality in $G^{\prime}$ which is violated by $\left(\bar{x}^{\prime}, \bar{y}^{\prime}\right)$.

Finally, suppose $\left(\bar{x}^{\prime}, \bar{y}^{\prime}\right)$ is obtained by $\theta_{5}$ with respect to two edges $u v$ and $v w$ with $\bar{x}_{u v}=\bar{x}_{v w}=1$ and $v$ with degree two. As the cut induced by $(S, i)$ is violated by $(\bar{x}, \bar{y})$, at most one of the edges $u v$ and $v w$ can be in $\delta(S)$. Suppose, without loss of generality, that $u v \in \delta(S), u \in S, v \in V \backslash S$ and $i \neq u$. Set $S^{\prime}=(S \backslash\{u\}) \cup\{\bar{v}\}$ and $i^{\prime}=i$ where $\bar{v}$ is the node that arises from the contraction of $u v$. We have $\bar{x}(\delta(S))+2 \sum_{j \in V \backslash S} \bar{y}_{i j}=$ $\bar{x}^{\prime}\left(\delta\left(S^{\prime}\right)\right)+2 \sum_{j \in V \backslash S^{\prime}} \bar{y}_{i j}^{\prime}<2$. Therefore, $\left(S^{\prime}, i^{\prime}\right)$ induces a violated cut inequality.

Conversely, let $S^{\prime} \subseteq V^{\prime}$ and $i^{\prime} \in S^{\prime}$ be such that the cut inequality induced by $\left(S^{\prime}, i^{\prime}\right)$ is violated by $\left(\bar{x}^{\prime}, \bar{y}^{\prime}\right)$. If $\left(\bar{x}^{\prime}, \bar{y}^{\prime}\right)$ is obtained by either $\theta_{1}, \theta_{2}$ or $\theta_{3}$ then by setting $S=S^{\prime}$ and $i=i^{\prime}$ we have that the cut induced by $(S, i)$ is violated by $(\bar{x}, \bar{y})$. 
Suppose $\left(\bar{x}^{\prime}, \bar{y}^{\prime}\right)$ is obtained by $\theta_{4}$ with respect to a node set $W \subseteq V \backslash\{0\}$. Let $w$ be the node arising from the contraction of $W$. If $w \in S^{\prime}$ and $i^{\prime} \neq w$ (resp. $i^{\prime}=w$ ), then let $S=$ $\left(S^{\prime} \backslash\{w\}\right) \cup W$ and $i=i^{\prime}$ (resp. $i=v$ for some $v \in W$ ).

If $w \notin S^{\prime}$, let $S=S^{\prime}$ and $i=i^{\prime}$.

In both cases, the cut induced by $(S, i)$ in $G$ is violated by $(\bar{x}, \bar{y})$.

Finally, suppose $\left(\bar{x}^{\prime}, \bar{y}^{\prime}\right)$ is obtained by $\theta_{5}$ with respect to two edges $u v$ and $v w$ such that $\bar{x}_{u v}=\bar{x}_{v w}=1$. Let $\bar{v}$ be the node arising from the contraction of $u v$. If $\bar{v} \notin S^{\prime}$, we can set $S=S^{\prime}$ and $i=i^{\prime}$. If $\bar{v} \in S^{\prime}$ and $i^{\prime}=\bar{v}$ (resp. $i \neq \bar{v}$ ) one can set $S=\left(S^{\prime} \backslash\{\bar{v}\}\right) \cup\{u\}$ and $i=u\left(\right.$ resp. $\left.i=i^{\prime}\right)$. In both cases, the cut induced by $(S, i)$ is violated by $(\bar{x}, \bar{y})$.

Before examining the extended $F$-partition (16) inequality, we give two lemmas.

Lemma 1. Let $\left(V_{0}, \ldots, V_{p}\right)$ be a partition and $e \in \delta\left(V_{0}\right)$ with $\bar{x}_{e}=1$. If there is a violated extended $F$-partition inequality (16) for this partition, then there is a violated extended $F$-partition inequality such that $e \in F$.

Proof. Suppose $\left(V_{0}, \ldots, V_{p}\right)$ induces a violated extended $F$-partition inequality, for some $F \subseteq \delta\left(V_{0}\right)$, that is, $\bar{x}\left(\delta\left(V_{0}, \ldots, V_{p}\right) \backslash F\right)+\sum_{l=1}^{p} \sum_{j \in V \backslash V_{l}} \bar{y}_{i_{i j}}<p-k$. If $e \in F$, then the lemma holds. So assume that $e \notin F$. Let $f$ be an edge of $F$. Exchanging $f$ by $e$ in $F$ yields an extended $F$-partition inequality with a violation not less than the initial one.

Lemma 2. Let $\left(V_{0}, \ldots, V_{p}\right)$ be a partition and $(u, w) \in A$ with $\bar{y}_{u w}=1$. If there is a violated extended $F$-partition inequality (16) for this partition, then there is a violated extended F-partition inequality such that $u \notin I=$ $\left\{i_{1}, \ldots, i_{p}\right\}$, i.e., $u$ is not a node fixed in a subset of the partition.

Proof. Suppose $\left(V_{0}, \ldots, V_{p}\right)$ induces a violated extended $F$-partition inequality, for some $F \subseteq \delta\left(V_{0}\right)$, that is, $\bar{x}\left(\delta\left(V_{0}, \ldots, V_{p}\right) \backslash F\right)+\sum_{l=1}^{p} \sum_{j \in V \backslash V_{l}} \bar{y}_{i_{i j}}<p-k$. If $u \notin I$, the lemma holds. So assume that $u \in I$. Without loss of generality, assume that $u=i_{1}$. If $w \in V_{1}$, then choosing $w$ as the fixed node of $V_{1}$, we obtain another violated extended $F$ partition inequality. So suppose $w \notin V_{1}$. If $V_{1} \backslash\{u\} \neq \emptyset$, then one can choose another node $k \in V_{1} \backslash\{u\}$ to be a fixed node in $V_{1}$. Note that the left hand side of the extended $F$-partition inequality will not increase as $\sum_{j \in V \backslash V_{1}} \bar{y}_{k j} \leq \sum_{j \in V \backslash V_{1}} \bar{y}_{u j}$. Hence $\left(V_{0}, \ldots, V_{p}\right), F$, and $(I \backslash\{u\}) \cup\{k\}$ yield another violated extended $F$-partition inequality. If $V_{1} \backslash\{u\}=\emptyset$, then we can put $V_{1}$ into $V_{0}$. As we know that $\bar{x}\left(\delta\left(V_{1}\right)\right)=0$ due to constraints (8), we have $\bar{x}\left(\delta\left(V_{0}, V_{2}, \ldots, V_{p}\right) \backslash F\right)+$ $\sum_{l=2}^{p} \sum_{j \in V \backslash V_{l}} \bar{y}_{i_{l} j}<p-k-1$. So this operation results in a violated extended $F$-partition inequality. So the lemma holds.

Theorem 16. There is an extended F-partition inequality (16) violated by $(\bar{x}, \bar{y})$ in $G$ if and only if there is an extended $F$-partition inequality violated by $\left(\bar{x}^{\prime}, \bar{y}^{\prime}\right)$ in $G^{\prime}$.
Proof. Let $\left(V_{0}, \ldots, V_{p}\right)$ be a partition denoted by $P_{1}$, $F \subset \delta\left(V_{0}\right)$ and $I=\left\{i_{1}, \ldots, i_{p}\right\}$ with $i_{l} \in V_{l}$ for $l=$ $1, \ldots, p$. Suppose that the extended $F$-partition inequality induced by $P_{1}, F$ and $I$ is violated by $(\bar{x}, \bar{y})$, that is to say $\bar{x}\left(\delta\left(V_{0}, \ldots, V_{p}\right) \backslash F\right)+\sum_{l=1}^{p} \sum_{j \in V \backslash V_{l}} \bar{y}_{i_{j} j}<p-k$. We will show that there is a partition of $V^{\prime}$, an edge set $F^{\prime}$ and a node set $I^{\prime}$ whose corresponding extended $F$-partition inequality in $G^{\prime}$ is violated by $\left(\bar{x}^{\prime}, \bar{y}^{\prime}\right)$. First observe that if $\bar{x}_{e}=0$ for some edge $e \in F$, and the cut inequalities are satisfied by $\bar{x}$, then the extended $F$-partition inequality cannot be violated by $\bar{x}$. Thus, we will suppose, without loss of generality, that $\bar{x}_{e}>0$ for all $e \in F$. If $\left(\bar{x}^{\prime}, \bar{y}^{\prime}\right)$ is obtained by operation $\theta_{1}$ with respect to an edge $e$ with $\bar{x}_{e}=0$ and $e \notin F$, then the same inequality is violated by $\left(\bar{x}^{\prime}, \bar{y}^{\prime}\right)$ in $G^{\prime}$. It is clear that if $\left(\bar{x}^{\prime}, \bar{y}^{\prime}\right)$ is obtained by operation $\theta_{2}$ with respect to an arc $a$ such that $\bar{y}_{a}=0$, then the same inequality is violated by $\left(\bar{x}^{\prime}, \bar{y}^{\prime}\right)$ in $G^{\prime}$. Suppose $\left(\bar{x}^{\prime}, \bar{y}^{\prime}\right)$ is obtained by $\theta_{3}$ with respect to an $\operatorname{arc}(u, w)$ with $\bar{y}_{u w}=1$. By Lemma 2 , we can assume that $u \notin I$. Let $u \in V_{k}$ for some $k \in\{0, \ldots, p\}$. For $l=0, \ldots p$, let $V_{l}^{\prime}=V_{l}$ if $k \neq l$ and $V_{l}^{\prime}=V_{l} \backslash\{u\}$ otherwise. It can be seen that $\left(V_{0}^{\prime}, \ldots, V_{p}^{\prime}\right), F$ and $I$ induce an extended $F$-partition inequality violated by $\left(\bar{x}^{\prime}, \bar{y}^{\prime}\right)$ in $G^{\prime}$.

Suppose $\left(\bar{x}^{\prime}, \bar{y}^{\prime}\right)$ is obtained by $\theta_{4}$ with respect to a node set $W \subset V$. Let $w$ be the node that arises from the contraction of $W$. Suppose first $W \subseteq V_{k}$ for some $k \in\{0, \ldots, p\}$. For $l=0, \ldots, p$, let $V_{l}^{\prime}=V_{l}$ if $k \neq l$ and $V_{l}^{\prime}=\left(V_{l} \backslash W\right) \cup\{w\}$ otherwise, and let $I^{\prime}=\left(I \backslash\left\{i_{k}\right\}\right) \cup\{w\}$ if $k>0$ and $I^{\prime}=$ $I$ otherwise. We can see that $\left(V_{0}^{\prime}, \ldots, V_{p}^{\prime}\right), F$ and $I^{\prime}$ induce an extended $F$-partition inequality violated by $\left(\bar{x}^{\prime}, \bar{y}^{\prime}\right)$ in $G^{\prime}$. Suppose now that $W$ is not a subset of a subset of the partition. Without loss of generality, assume that $W \cap V_{i} \neq \emptyset$ for $i=$ $1, \ldots, k$. Because $G(W)$ is 2-edge connected and $\bar{x}_{e}=1$ for all $e \in E(W), \bar{x}\left(\delta\left(V_{1}, \ldots, V_{k}\right)\right) \geq k$. So we can contract $V_{1}, \ldots, V_{k}$, and choose a new fixed node for the resulting set. In this case, the right-hand side of the inequality reduces by $k-1$, whereas the left-hand side decreases by at least $k$, which yields an extended $F$-partition inequality violated by $\left(\bar{x}^{\prime}, \bar{y}^{\prime}\right)$ in $G^{\prime}$. If $W \cap V_{0} \neq \emptyset$, then by Lemma 1 , we can assume that every $e \in \delta(W) \cap \delta\left(V_{0}\right)$ is also in $F$, implying that $|F \cap E(W)| \geq 2$. Note that $\bar{x}\left(\delta\left(V_{1}, \ldots, V_{k}\right)\right) \geq k-1$. Let $V_{0}^{\prime}=\cup_{i=0}^{k} V_{i}, F^{\prime}=F \cap \delta\left(V_{0}^{\prime}\right)$, and $I^{\prime}=I \backslash\left\{i_{1}, \ldots, i_{k}\right\}$. Note that $\left|F^{\prime}\right| \leq|F|-2$. It is not hard to see that the extended $F$-partition inequality induced by $\left(V_{0}^{\prime}, V_{k+1}, \ldots, V_{p}\right), F^{\prime}$, and $I^{\prime}$ is violated by $\left(\bar{x}^{\prime}, \bar{y}^{\prime}\right)$ in $G^{\prime}$.

Finally, suppose $\left(\bar{x}^{\prime}, \bar{y}^{\prime}\right)$ is obtained by $\theta_{5}$ with respect to two edges $u v$ and $v w$ with $\bar{x}_{u v}=\bar{x}_{v w}=1$ and $v$ with degree two. Let $\bar{v}$ be the node that arises from the contraction of $u v$. Suppose $u v \in E\left(V_{k}\right)$ for some $k \in\{0, \ldots, p\}$ (the case $v w \in E\left(V_{k}\right)$ is similar). For $l=0, \ldots, p$, let $V_{l}^{\prime}=V_{l}$ if $k \neq l$ and $V_{l}^{\prime}=\left(V_{l} \backslash\{u, v\}\right) \cup\{\bar{v}\}$ otherwise and let $I^{\prime}=$ $\left(I \backslash\left\{i_{k}\right\}\right) \cup\{\bar{v}\}$ if $k>0$ and $I^{\prime}=I$ otherwise. It is easily seen that $\left(V_{0}^{\prime}, \ldots, V_{p}^{\prime}\right), F$ and $I^{\prime}$ induce an extended $F$-partition inequality violated by $\left(\bar{x}^{\prime}, \bar{y}^{\prime}\right)$ in $G^{\prime}$. If $u, v, w$ are in different subsets or if $u, w \in V_{j}$ and $v \in V_{k}$ with $j \neq k$, then by contracting the subsets intersecting $\{u, v, k\}$ and choosing a new fixed node for the new set we obtain a violated extended 
$F$-partition inequality. Therefore we do not need to consider such cases.

Conversely, let $\left(V_{0}^{\prime}, \ldots, V_{p}^{\prime}\right)$ be a partition of $V^{\prime}$ denoted by $P_{1}^{\prime}, F^{\prime} \subset \delta\left(V_{0}^{\prime}\right)$ and $I^{\prime}=\left\{i_{1}^{\prime}, \ldots, i_{p}^{\prime}\right\}$ with $i_{l}^{\prime} \in V_{l}^{\prime}$ for $l=1, \ldots, p$. Suppose that the extended $F$-partition inequality induced by $P_{1}^{\prime}, F^{\prime}$ and $I^{\prime}$ is violated by $\left(\bar{x}^{\prime}, \bar{y}^{\prime}\right)$, that is to say $\bar{x}^{\prime}\left(\delta\left(V_{0}^{\prime}, \ldots, V_{p}^{\prime}\right) \backslash F^{\prime}\right)+\sum_{l=1}^{p} \sum_{j \in V^{\prime} \backslash V_{l}^{\prime}} \bar{y}_{i_{l}^{\prime} j}^{\prime}<p-k$. If $\left(\bar{x}^{\prime}, \bar{y}^{\prime}\right)$ is obtained by either $\theta_{1}$ or $\theta_{2}$ then $P_{1}^{\prime}, F^{\prime}$, and $I^{\prime}$ also induce an extended $F$-partition inequality violated by $\bar{x}, \bar{y}$ in $G$. If $\left(\bar{x}^{\prime}, \bar{y}^{\prime}\right)$ is obtained by $\theta_{3}$ with respect to an $\operatorname{arc}(i, j)$ then $\left(V_{0}^{\prime} \cup\{i\}, V_{1}^{\prime}, \ldots, V_{p}^{\prime}\right), F^{\prime}$ and $I^{\prime}$ also induce an extended $F$-partition inequality violated by $(\bar{x}, \bar{y})$ in $G$.

Suppose $\left(\bar{x}^{\prime}, \bar{y}^{\prime}\right)$ is obtained by $\theta_{4}$ with respect to a node set $W$. Let $w$ be the node arising from the contraction of $W$ and assume that $w \in V_{k}^{\prime}$ for some $k \in\{0, \ldots, p\}$. Then $\left(V_{0}, \ldots, V_{p}^{\prime}\right), F^{\prime}$ and $I^{\prime}$ induce an extended $F$-partition inequality violated by $(\bar{x}, \bar{y})$ in $G$, where $V_{i}=V_{i}^{\prime}$ if $i \neq k$, and $V_{i}=\left(V_{i}^{\prime} \backslash\{w\}\right) \cup W$ otherwise.

Finally, suppose $\left(\bar{x}^{\prime}, \bar{y}^{\prime}\right)$ is obtained by $\theta_{5}$ with respect to two edges $u v$ and $v w$ such that $\bar{x}_{u v}=\bar{x}_{v w}=1$. Let $\bar{v}$ be the node arising from the contraction of $u v$ and assume that $\bar{v} \in V_{k}^{\prime}$ for some $k \in\{0, \ldots, p\}$. Then $\left(V_{0}, \ldots, V_{p}\right), F^{\prime}$ and $I^{\prime}$ induce an extended $F$-partition inequality violated by $\bar{x}, \bar{y}$ in $G$, where $V_{i}=V_{i}^{\prime}$ if $i \neq k$, and $V_{i}=\left(V_{i}^{\prime} \backslash\{\bar{v}\}\right) \cup\{u, v\}$ otherwise.

We turn now our attention to the star-path inequalities (19). We first give a lemma.

Lemma 3. An ordered set I cannot yield a violated star-path (19) inequality if there is an edge $e \in P_{I}$ with $\bar{x}_{e}=1$.

Proof. Let $I=\left\{i_{0}, \ldots, i_{m}\right\}$ and $e=\left\{i_{l}, i_{l}+1\right\}$. Assume that $e \in P_{I}$ and $\bar{x}_{e}=1$. This implies that $y_{i_{l} j}=0$ for all $j \in V \backslash\left\{i_{l}\right\}$ and $y_{i_{l+1} j}=0$ for all $j \in V \backslash\left\{i_{l+1}\right\}$. Hence, $\sum_{j \in V \backslash\left\{i_{i}\right\}} y_{i_{l} j}+\sum_{j \in V \backslash\left\{i_{l+1}\right\}} y_{i_{l+1} j}=0$. As $(\bar{x}, \bar{y})$ satisfies inequalities (8) the following hold:

$$
\begin{aligned}
& x_{i_{0} i_{1}}+\sum_{j=1}^{m} y_{i_{0} i_{j}} \leq x_{i_{0} i_{1}}+y_{i_{1} i_{0}}+\sum_{k \in V \backslash\left\{i_{0}\right\}} y_{i_{0} k} \leq 1 \\
& \vdots \\
& x_{i_{l-1} i_{l}}+\sum_{j \in V \backslash\left\{i_{l-1}\right\}} y_{i_{l-1} j} \leq x_{i_{l-1} i_{l}}+y_{i_{l} i_{l-1}}+\sum_{j \in V \backslash\left\{i_{l-1}\right\}} y_{i_{l-1} j} \leq 1 \\
& x_{i_{l} i_{l+1}}+\sum_{j \in V \backslash\left\{i_{l}\right\}} y_{i_{l} j}+\sum_{j \in V \backslash\left\{i_{l+1}\right\}} y_{i_{l+1} j}=1 \\
& x_{i_{l+1} i_{l+2}}+\sum_{j \in V \backslash\left\{i_{l+2}\right\}} y_{i_{l+2} j} \leq x_{i_{l+1} i_{l+2}}+y_{i_{l+1} i_{l+2}} \sum_{j \in V \backslash\left\{i_{l+2}\right\}} y_{i_{l+2} j} \leq 1
\end{aligned}
$$

$$
x_{i_{m-1} i_{m}}+\sum_{j \in V \backslash\left\{i_{m}\right\}} y_{i_{m} j} \leq x_{i_{m-1} i_{m}}+y_{i_{m-1} i_{m}} \sum_{j \in V \backslash\left\{i_{m}\right\}} y_{i_{m} j} \leq 1
$$

Note that we obtain the expressions on the left hand side by omitting some of the terms of the left hand sides of inequalities (8). Summing them we obtain $x\left(P_{I}\right)+$ $\sum_{i \in I \backslash\left\{i_{0}\right\}} \sum_{(i, j) \in A} y_{i j}+\sum_{j \in I:\left(i_{0}, j\right) \in A} y_{i_{0} j} \leq m$ which is the starpath inequality induced by $I$, showing that the inequality is not violated.

Theorem 17. There is a star-path inequality (19) violated by $(\bar{x}, \bar{y})$ in $G$ if and only if there is a star-path inequality violated by $\left(\bar{x}^{\prime}, \bar{y}^{\prime}\right)$ in $G^{\prime}$.

Proof. Let $I=\left\{i_{0}, \ldots, i_{m}\right\}$ be an ordered set inducing a star path inequality violated by $(\bar{x}, \bar{y})$. First, it is clear that if $\left(\bar{x}^{\prime}, \bar{y}^{\prime}\right)$ is obtained by either $\theta_{1}$ or $\theta_{2}$, then $I$ still induces a star path inequality violated by $\left(\bar{x}^{\prime}, \bar{y}^{\prime}\right)$ in $G^{\prime}$. Also by Lemma 3 , if $\bar{x}_{e}=1$, then $e \notin P_{I}$. Thus $\theta_{4}$ and $\theta_{5}$ do not affect $I$ and hence the star path inequality induced by $I$ remains violated by $\left(\bar{x}^{\prime}, \bar{y}^{\prime}\right)$ in $G^{\prime}$. Now, suppose $\bar{y}_{u v}=1$ for some $(u, v) \in A$. Without loss of generality, we may assume that $u=i_{l} \in I$. Hence, $\bar{y}_{i_{l} k}^{\prime}=0$ for all $k \in V \backslash\left\{i_{l}, v\right\}$, and $\bar{x}_{i_{l} k}^{\prime}=0$ for all $k \in$ $V \backslash\left\{i_{l}\right\}$. As the star-path inequality induced by $I$ is violated, $z=\bar{x}\left(P_{I}\right)+\sum_{i \in I \backslash\left\{i_{0}\right\}} \sum_{(i, j) \in A} \bar{y}_{i j}+\sum_{j \in I:\left(i_{0}, j\right) \in A} \bar{y}_{i_{0} j}>m$. Let $I^{\prime}=I \backslash\left\{i_{l}\right\}$. To obtain the star-path inequality induced by $I^{\prime}$ from the one induced by $I$, we need to remove the terms related to node $i_{l}$ from the left-hand side and add $x_{i_{l-1} i_{l+1}}$. Decreasing the right-hand side by one we obtain the new starpath inequality induced by $I^{\prime}$. Because $z-1+\bar{x}_{i_{l-1}, i_{l+1}}>m-1$, this new star-path inequality is also violated by $\left(\bar{x}^{\prime}, \bar{y}^{\prime}\right)$. So there is a violated star-path inequality in $G^{\prime}$ after reduction by $\theta_{3}$, if there is one in $G$.

The converse of the theorem can be easily seen to be true.

\section{SEPARATION ALGORITHMS}

In this section, we shall describe our separation algorithms. For a given fractional solution $\left(x^{*}, y^{*}\right)$, we define the following sets $V_{h}=\left\{i \in V: \sum_{j \in V \backslash\{i\}} y_{i j}^{*}=0\right\} \cup\{0\}, V_{p h}=$ $\left\{i \in V: \sum_{j \in V \backslash\{i\}} y_{i j}^{*}>0\right.$ and $\left.x^{*}(\delta(i))>0\right\}, V_{u}=\{i \in$ $V: x^{*}(\delta(i))=0$ and $\exists j \in V \backslash\{i\}$ such that $\left.y_{i j}^{*}=1\right\}$ and $V_{p u}=\left\{i \in V \backslash V_{u}: x^{*}(\delta(i))=0\right\}$. We call the elements of these sets hubs, partial hubs, users, and partial users, respectively. Note that $V_{\mathrm{h}}, V_{\mathrm{ph}}, V_{\mathrm{u}}, V_{\mathrm{pu}}$ form a partition of $V$. We also define $V^{*}=V_{\mathrm{h}} \cup V_{\mathrm{ph}}, E^{*}=\left\{\{i, j\} \in E: x_{i j}^{*}>0\right\}$ and $A^{*}=\left\{(i, j) \in A: 0<y_{i j}^{*}<1\right\} . G^{*}=\left(V^{*}, E^{*}\right)$ is our support graph and it may be disconnected. Let $G^{i}=\left(V^{i}, E^{i}\right)$ for $i=0, \ldots, r$ be the $i$ th connected component of $G^{*}$. Without loss of generality, we assume $0 \in V^{0}$. Clearly $G^{0}=G^{*}$ if $G^{*}$ is connected. Violated clique inequalities (8) are found by complete enumeration and for the cut (10), star-path (19) and extended $F$-partition inequalities (16) the separation algorithms are described in the following sections. We want to note that all the separation procedures described are performed on the graphs obtained by the reduction operations unless otherwise specified. Then the inequalities found by the separation algorithms are lifted as described below to obtain the inequalities to be added to the model. Let $\bar{W}(\bar{V})$ be the set of nodes that arises from $\theta_{4}\left(\theta_{5}\right)$ and $|\bar{W}|=q(|\bar{V}|=r)$. Let $W_{i}$ be the contracted node set and $w_{i}$ the corresponding 
node for $i=1, \ldots, q$. Similarly, let $u_{i} v_{i}$ be the contracted edge and $\bar{v}_{i}$ be the corresponding node for $i=1, \ldots, r$.

Let $\left(S^{\prime}, i^{\prime}\right)$ be a pair inducing a violated cut inequality (10) in $G^{\prime}$. Without loss of generality, say $w_{1}, \ldots, w_{j} \in S^{\prime}$ for some $j \in\{1, \ldots, q\}$ and $\bar{v}_{1}, \ldots, \bar{v}_{k} \in S^{\prime}$ for some $k \in\{1, \ldots, r\}$. Let $S=S^{\prime} \backslash\left(\cup_{l=1}^{j} w_{l} \cup_{l=1}^{k} \bar{v}_{l}\right) \cup\left(\cup_{l=1}^{j} W_{l} \cup_{l=1}^{k} \bar{V}_{l}\right)$ and

$$
i= \begin{cases}i^{\prime} & \text { if } \quad i^{\prime} \notin \bar{W} \cup \bar{V}, \\ v_{l} & \text { if } i^{\prime}=\bar{v}_{l} \text { for some } l=1, \ldots, k, \\ \text { any node in } W_{l} & \text { if } \quad i^{\prime}=w_{l} \text { for some } l=1, \ldots, j .\end{cases}
$$

Then $(S, i)$ induce a violated cut inequality in $G$.

For extended $F$-partition inequalities (16) we define $Y$ to be the set of nodes deleted by $\theta_{3}$. Let $\left(V_{0}^{\prime}, \ldots, V_{p}^{\prime}\right)$, $F^{\prime}$ and $i_{1}^{\prime}, \ldots, i_{p}^{\prime}$ define a violated extended $F$-partition inequality in $G^{\prime}$. Set $V_{i}=V_{i}^{\prime} \backslash\left(\cup_{1 \leq l \leq q: w_{l} \in V_{i}^{\prime}}\left\{w_{l}\right\} \cup_{1 \leq l \leq r: \bar{v}_{l} \in V_{i}^{\prime}}\right.$ $\left.\left\{\bar{v}_{l}\right\}\right) \cup\left(\cup_{1 \leq l \leq q: w_{l} \in V_{i}^{\prime}} W_{l} \cup_{1 \leq l \leq r: \bar{v}_{l} \in V_{i}^{\prime}} \bar{V}_{l}\right)$ for $i=1, \ldots, p$ and $V_{0}=V_{0}^{\prime} \backslash\left(\cup_{1 \leq l \leq q: w_{l} \in V_{0}^{\prime}}\left\{w_{l}\right\} \quad \cup_{1 \leq l \leq r: \bar{v}_{l} \in V_{0}^{\prime}}\left\{\bar{v}_{l}\right\}\right) \cup$ $\left(\cup_{1 \leq l \leq q: w_{l} \in V_{0}^{\prime}} W_{l} \cup_{1 \leq l \leq r: \bar{v}_{l} \in V_{0}^{\prime}} \bar{V}_{l}\right) \cup Y$. The fixed nodes should also be updated as follows:

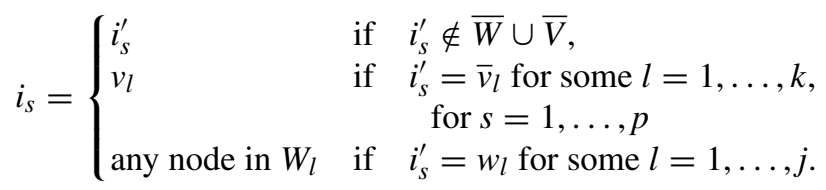

Then $\left(V_{0}, \ldots, V_{p}\right), F^{\prime}$ and $i_{1}, \ldots, i_{p}$ induce a violated extended $F$-partition inequality (16) in $G$.

For the star-path inequalities as the reduction operations do not affect the ordered set of nodes defining the violated star-path inequality, the ordering can also be used in $G$. Now we can give our separation algorithms for each class of valid inequalities.

\subsection{Cut Inequalities}

A cut inequality (10) is defined by a node set $S \subseteq V \backslash\{0\}$ and a fixed node $i \in S$. For a given $i \in V \backslash\{0\}$, it is possible to check if there exists a subset $S \subseteq V \backslash\{0\}$ with $i \in S$ for which the cut inequality is violated by solving a minimum cut problem. Let $G_{i}^{*}=\left(V^{*} \cup\{i\}, E_{i}^{*}\right)$ where $E_{i}^{*}=E^{*} \cup\{\{i, j\}$ : $j \in V^{*}$ and $\left.(i, j) \in A^{*}\right\}$. Set the capacity of edge $e$ to $x_{e}^{*}$ if $i \notin e$, and to $x_{i j}^{*}+2 y_{i j}^{*}$ otherwise. Labbé et al. [21] showed that a maximum violated cut inequality with fixed node $i$ can be found by solving a minimum cut problem on this graph separating nodes $i$ and 0 . If the minimum cut capacity is less than 2 , then there is a violated cut inequality. Therefore cut inequalities can be separated exactly by solving $|V|-1$ minimum cut problems. We also use the separation method of [21] together with a heuristic algorithm to speed up the separation.

Our separation algorithm works in three phases. We first use the connected components of the support graph to generate violated cut inequalities. For a given connected component $G^{i}=\left(V^{i}, E^{i}\right)$ we compute the violation of the cut inequality defined by every $j \in V^{i}$ and node set $V^{i} \cup\left\{k \in V_{\mathrm{u}} \cup V_{\mathrm{ph}}: y_{j k}^{*}>0\right\}$. As $x^{*}\left(\delta\left(V^{i}\right)\right)=0$, it is very likely that we find a violated inequality this way. The most violated cut inequality is selected for $i=1, \ldots, r$. If $r>1$ we perform the same operations for $\cup_{i=1}^{q} V^{i}$ for $q=2, \ldots, r$.

Second, we use our heuristic on $G^{0}$. The heuristic is based on the algorithm of Hao and Orlin [15] which finds a global minimum cut, i.e., a cut with the minimum capacity among all cuts of a graph. In this algorithm, $n-1$ minimum cut problems, where $n$ is the number of nodes of the graph, are solved. Let $s_{i}$ be the source node of the $i$ th minimum cut problem and $t$ the sink node at the beginning. In the $i$ th step, the minimum cut between $s_{i}$ and $\left\{t, s_{1}, \ldots, s_{i-1}\right\}$ is found. Hao and Orlin [15] show that the cut with the minimum capacity among the ones found in the algorithm is the global minimum cut of the graph. Moreover, they select the source nodes in such a way that the running time of the algorithm is equivalent to that of a single minimum cut problem. We apply their algorithm on $G^{0}$ with the capacity of each edge $e \in E^{0}$ being equal to $x_{e}^{*}$. Our root node is the initial sink for the algorithm. Using this we obtain $\left|V^{0}\right|-1$ cut sets, say $S_{1}, \ldots, S_{\left|V^{0}\right|-1}$. Since $y^{*}$ values are ignored in edge capacities, there are three possible outcomes for every cutset. If the capacity of the cut is greater than or equal to 2 then there is no violated cut inequality associated with this cut. If the capacity is less than 2 , then the violation must be calculated by taking $y^{*}$ values into account for a given fixed node to see if the corresponding cut inequality is really violated. Therefore, for $i=1, \ldots,\left|V^{0}\right|-1$ such that the capacity of $\left[S_{i}, V^{0} \backslash S_{i}\right]$ is less than 2 , we calculate the violation of the cut inequality defined by $S_{i}$ and $j$ for every $j \in S_{i} \cup V_{\mathrm{u}} \cup V_{\mathrm{ph}}$. If there is at least one violated cut inequality, we choose the one with the maximum violation. If there is more than one fixed node with the same violation, then we choose the one which is used less often in previous cut inequalities. We can keep track of this information using an array. If there is still a tie, we break it arbitrarily. Note that if the fixed node, say $j \in V_{\mathrm{u}} \cup V_{\mathrm{pu}}$, is chosen for a given cutset $S$, the cutset must be extended as $S \cup\{j\}$ since $j \notin S$.

There are two advantages of this method. First, it is very fast. Second, if the minimum cut capacities are all greater than or equal to 2 , then we can conclude that there is no violated cut inequality. Notice that we are not interested in the nodes of $V_{\mathrm{u}} \cup V_{\mathrm{pu}}$ in minimum cut computations as there is no adjacent edge to them in the support graph. These nodes are put together with node 0 . But if the fixed node of a given cut inequality is assigned to a user or partial user node $j$ then we move it from $V \backslash S$ to $S$. This operation does not affect the first term of the cut inequality but reduces the second term and hence the violation increases.

As finding a minimum cut between $i$ and 0 provides the most violated cut inequality with fixed node $i$, we need to solve minimum cut problems for every node of the backbone network except for the root node. This is necessary as the cut inequalities are in the model formulation and must be separated exactly. However, as we use a heuristic step first, we can eliminate some nodes from consideration in the exact separation phase. This is possible because either some violated cut inequality is found for the fixed node in the heuristic phase or 
from the information obtained from the first step we expect that there is no violated cut inequality with this fixed node. So we define $C$ to be the set of nodes $i$ in $V_{\text {pu }} \cup V_{\text {ph }}$ such that $i$ is not used as a fixed node in the inequalities added by the heuristic. The nodes of $C$ will be referred to as candidate nodes and are the nodes which are not eliminated from consideration after the heuristic phase. We remark that nodes in $V_{\mathrm{h}} \cup V_{\mathrm{u}}$ are excluded from this candidate list due to the following reasons. Let $i \in V_{\mathrm{h}}$ and $S \subseteq V \backslash\{0\}$ be such that $i \in S$. If $x^{*}(\delta(S))<2$, then there is a violated cut inequality and $i$ gives the maximum violation as $\sum_{j \in V \backslash S} y_{i j}^{*}=0$. So if a violated cut inequality with fixed node $i$ is not found in the heuristic phase we can say that either there is no violated cut inequality with fixed node $i$ or another inequality with the same cutset but with a different fixed node is found. Therefore, we do not include hubs in $C$. A similar reasoning can be done for the users. Besides, there is no guarantee we will find a different cut for this node by using the exact separation if we find a violated cut inequality for some $i$ in the heuristic phase. So we do not include such nodes in $C$, either.

If at least one cut with capacity less than 2 is found in the heuristic phase and $C \neq \varnothing$ we pass to the last step, the exact separation phase. In this phase we solve a minimum cut problem between $i$ and 0 on $G_{i}^{*}$ for every $i \in C$.

The Goldberg-Tarjan algorithm [13] is used to solve the minimum cut problems. Our separation procedure is given in Algorithm 1. As it turns out during our experimentation, the heuristic part significantly improves the CPU time of our branch-and-cut algorithm.

\subsection{Extended F-Partition Inequalities}

We use two heuristic methods to find violated extended $F$ partition inequalities (16). The first one is based on searching odd fractional cycles in $G^{*}$. A set of nodes $\left\{v_{1}, \ldots, v_{p}\right\}$ which induces an odd cycle is determined, if one exists. Let $V_{0}=$ $V \backslash\left\{v_{1}, \ldots, v_{p}\right\}$. The edges in $\delta\left(V_{0}\right)$ with values greater than $\frac{1}{2}$ are included in $F$ in such a way that $|F|$ becomes odd. The corresponding inequality is checked for violation.

The second heuristic is based on finding a maximum cut in a graph. Because the maximum cut problem is NP-hard, it is solved heuristically as follows. We associate with each edge $e$ a capacity equal to $1-x_{e}^{*}$. We use the algorithm of Hao and Orlin [15] on $G^{0}$ to find $\left|V^{0}\right|-1$ minimum cuts. Let $S$ be a cutset obtained by this algorithm such that $0 \in S$ and $V_{0}=S \cup V_{\mathrm{u}} \cup V_{\mathrm{pu}}$. Let $V^{0} \backslash S=\left\{v_{1}, \ldots, v_{p}\right\}$. Then our partition is $\left(V_{0}, V^{1}, \ldots, V^{r}, v_{1}, \ldots, v_{p}\right)$. We construct $F$ as we did in the first heuristic. For the strong component $G^{i}$, node $j \in V^{i}$ with the largest $\sum_{k \in V \backslash V^{i}} y_{j k}^{*}$ value is selected as the fixed node for $i=1, \ldots, r$. We check the extended $F$ partition inequality induced by this partition and fixed nodes to see if it is violated.

Note that we included the connected components as separate node sets while forming the partition. This is because doing so increases the violation of a given extended $F$ partition inequality. Consider a connected component $G^{i}$.

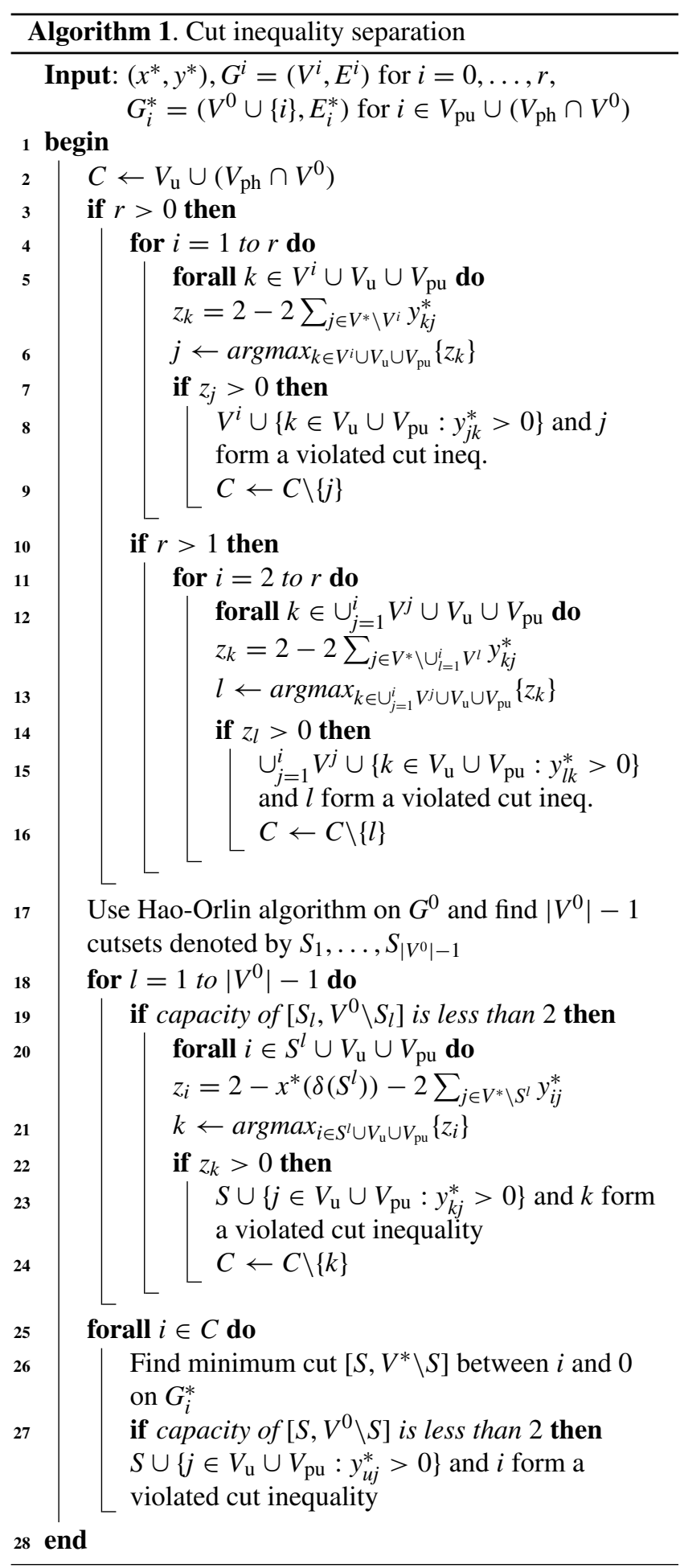

Because $x^{*}\left(\delta\left(V^{i}\right)\right)=0$ and $\sum_{k \in V \backslash V^{i}} y_{j k}^{*} \leq 1$ for any fixed node $j \in V^{i}$, the increase in the left hand side of the inequality is at most 1 . But the right-hand side increases exactly by one when we include the connected component in the partition. Therefore, using a connected component, we can increase the violation of a given extended $F$-partition inequality. 


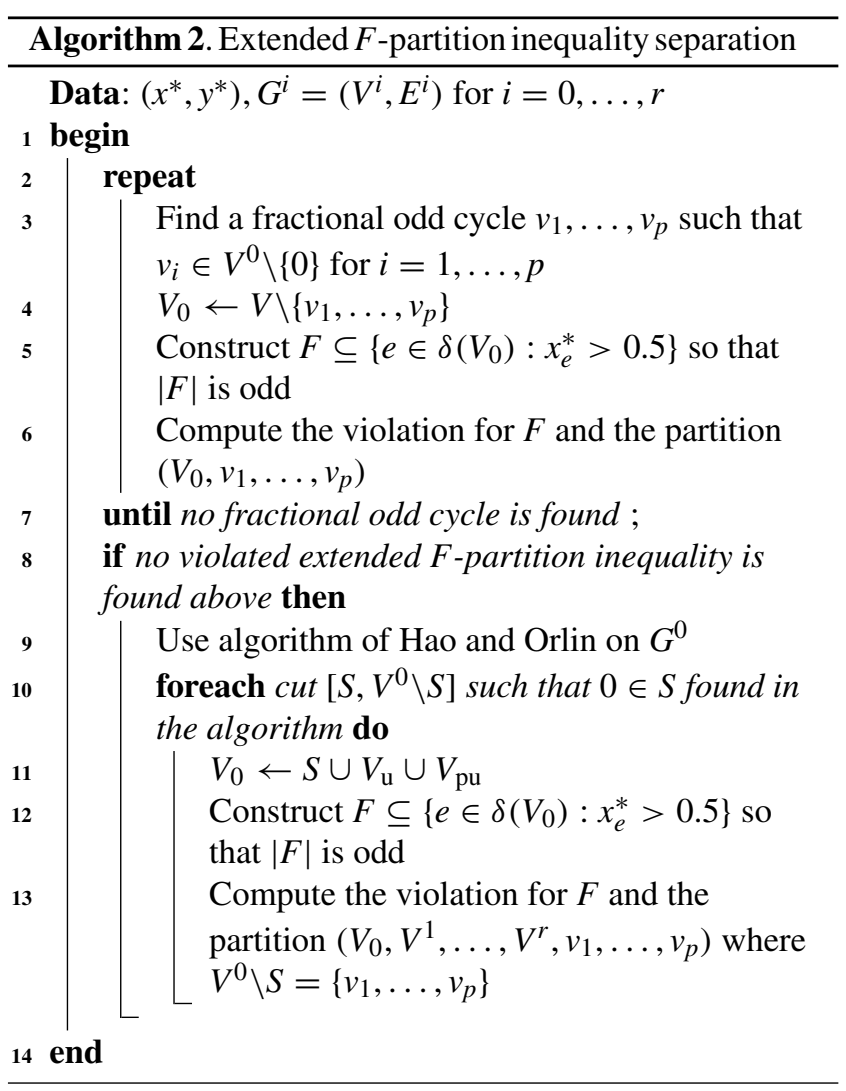

\subsection{Star-Path Inequalities}

Because the separation problem for the star-path inequalities (19) is NP-complete, we developed a heuristic algorithm. Assuming that all clique inequalities (8) are satisfied, it can be seen that a star-path inequality can be violated only if the $x$ terms of the inequality are positive. Moreover, we observed that violated star-path inequalities are frequently induced by paths which include nodes to which the initial node $s$ is assigned. For this reason we restrict our search for paths on these nodes. For some initial node $s$, let $V_{s}=\left\{i \in V^{*} \backslash\{0\}\right.$ : $\left.y_{s i}^{*}>0\right\} \cup\{s\}$ and $E_{s}=\left\{i j: i \in V_{s}, j \in V_{s}, x_{i j}^{*}<1\right\}$. Note that we omitted the edges with values 1 by Lemma 3 . The edges of $E_{s}$ are ordered in a decreasing way and edges are selected from this list until a simple path starting at node $s$ and covering all nodes of $V_{s}$ is obtained. After finding such a path we analyze the nodes to see if their removal increases the amount of violation of the inequality. We restrict the heuristic to the search of star-path inequalities with more than 4 nodes as the 3 and 4 node star-path inequalities are generated by enumeration. Using the necessary conditions stated in Lemma 3 for a star-path inequality to be violated, the enumeration can be performed very efficiently.

\section{COMPUTATIONAL RESULTS}

Based on our polyhedral analysis and the separation algorithms described earlier, we developed a branch-and-cut

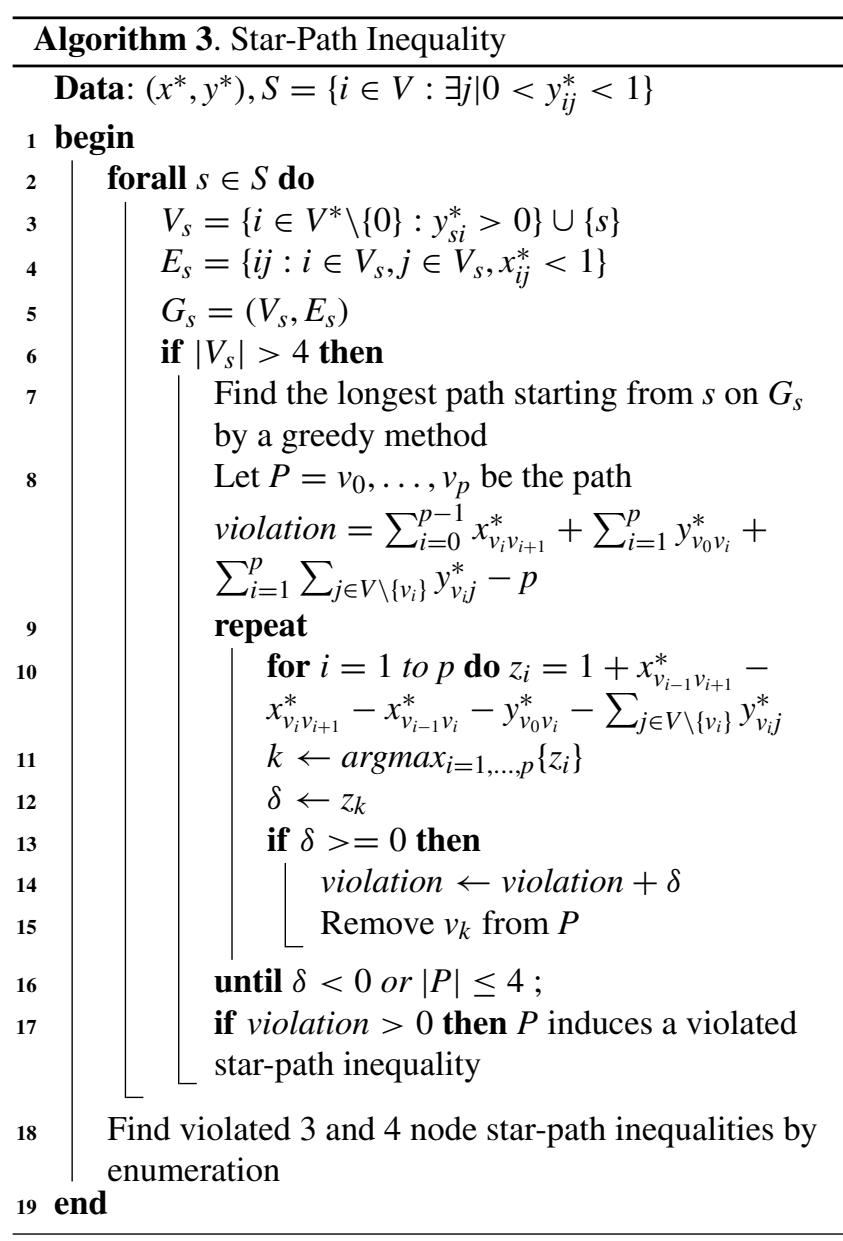

algorithm for the 2ECSSP. We start the optimization by solving the following linear program:

$$
\begin{aligned}
& \min \sum_{i j \in E} c_{i j} x_{i j}+\sum_{(i, j) \in A} d_{i j}^{\prime} y_{i j} \\
& \text { s.t. } \\
& \quad x_{0 i}+\sum_{j \in V \backslash\{i\}} y_{i j} \leq 1 \quad \forall i \in V \backslash\{0\} \\
& x(\delta(i))+\sum_{j \in V \backslash\{i\}} y_{i j} \geq 2 \quad \forall i \in V \backslash\{0\} \\
& 0 \leq x_{i j} \leq 1 \quad \forall i j \in E \\
& 0 \leq y_{i j} \leq 1 \quad \forall(i, j) \in A
\end{aligned}
$$

The solution $(\bar{x}, \bar{y})$ of this initial subproblem is feasible for 2ECSSP if it satisfies the cut inequalities, the clique inequalities and integrality constraints. Therefore, at each iteration of the branch-and-cut algorithm, we solve the separation problems to determine if there are violated inequalities. The different inequalities are separated in the following order: clique (8), cut (10), F-partition (16), and star-path (19) inequalities. We generate up to 200 violated valid inequalities at each iteration and look for a violated inequality only if we cannot find a violated inequality of a previous class 
and found less than 200 inequalities. Note that if a solution is integral and there is no violated cut or clique inequality, then we do not need to solve the separation problems for the $F$-partition and the star-path inequalities.

We adapt the instance generation method of Labbé et al. [21]. Our test problems are based on TSP instances from TSPLIB 2.1 [29]. To compute the backbone link setup costs and assignment costs we use the following formulas, $c_{i j}=$ $\left\lceil\alpha l_{i j}\right\rceil$ and $d_{i j}=\left\lceil(10-\alpha) l_{i j}\right\rceil$ where $l_{i j}$ denotes the distance between nodes $i$ and $j$ in the TSPLIB instances and $\alpha \in$ $\{3,5,7,9\}$. We set $d_{i i}=0$ for all $i \in V \backslash\{0\}$. Note that as $\alpha$ decreases, assignment costs increase, whereas backbone link setup costs decrease, i.e., the problem gets closer to the 2-edge connected subgraph problem. Conversely, as $\alpha$ increases the number of nodes chosen to be hubs decreases and the problem gets farther from the 2-edge connected subgraph problem.

A construction heuristic is used to find an initial solution at the beginning of the algorithm. We start from node 0 and apply the nearest neighbor TSP heuristic to obtain a cycle that includes all nodes of the graph. As this cycle is 2edge connected, it forms a feasible solution. Throughout the branch-and-cut algorithm, we also use an LP based improvement heuristic. The edges are ranked in a decreasing order according to their values in the fractional solution. We start with an empty set and add the edges one by one until we obtain a cycle. The remaining nodes which are not included in the cycle are assigned to a node in the cycle with minimum assignment cost.

We implemented our algorithm in $\mathrm{C}++$ using ABACUS 3.0 as the framework and CPLEX 11.0 as the LP solver. Computational analysis is performed on a workstation with $2.66 \mathrm{GHz}$ xeon processor and $8 \mathrm{~Gb}$ of ram. We use best first search as the search strategy and strong branching as the branching strategy. Tailing off control is used; we branch if the improvement in the objective function value is small in 10 subsequent iterations. We do not use pool separation, and added inequalities are removed if they are not active in 5 subsequent iterations.

Our computational results are provided in Tables 1-6. In all the tables, the first two columns denote the name of the instance and the $\alpha$ value, respectively. The number of nodes in the instance is also included in the name of the instance. The other columns given in the tables are explained below:

NClq, number of generated clique inequalities;

NCut, number of generated cut inequalities;

NFP, number of generated $F$-partition inequalities;

NSP, number of generated star-path inequalities;

Opt, optimal objective function value;

NNode, number of branch-and-cut nodes evaluated;

$\mathrm{Cpu}$, total CPU time in seconds (rounded to the nearest integer);

Gap1, the relative error between the optimal solution value and the lower bound obtained at the root node of the branch-and-cut tree;

Gap2, the relative error between the best upper bound (the optimal solution value if the problem is solved to optimality) and the lower bound obtained at the end of the branch-and-cut algorithm.
In Table 1, only clique and cut inequalities are used in solving the instances. In Table 2, we present the results obtained using extended $F$-partition inequalities together with the basic inequalities. The last three columns show the improvement in the gap, CPU time, and the number of branchand-cut nodes attained by adding the extended $F$-partition inequalities, respectively. Here, a negative value means the performance gets worse after adding the extended $F$-partition inequalities. If an instance is not solved to optimality using only clique and cut inequalities, then it is not possible to compute the improvement for CPU time; we denote such cases with **. Table 3 includes the results obtained using both extended $F$-partition and star-path inequalities together with the basic inequalities, and its columns are similar to those of Table 2 except that the improvement columns give the comparisons between Tables 2 and 3. Finally, the instances are solved with all the valid inequalities without using the reduction operations and the results are given in Table 4. The last three columns of this table present information about comparisons made between Tables 3 and 4, and a positive value indicates that the algorithm performs better with reduction operations.

We solved 28 instances, 10 of which reached optimality at the root node. We observe that the number of branch-and-cut nodes exploited tends to decrease as $\alpha$ increases. The optimal solution is found at the root node when $\alpha=9$ even if the $F$-partition and star-path inequalities are not used. However, when $\alpha=3$ and no $F$-partition and star-path inequalities are used, the number of nodes increases to an extent that the algorithm terminates before reaching the optimal solution due to memory problems. When only the clique and cut inequalities are used, 8 instances could not be solved to optimality due to memory problems and these are denoted with $*$ in the CPU column of Table 1. For such instances, Gap2 could be as large as $23 \%$ and the average Gap2 is around $8.75 \%$. The average Gap 1 is $0.41 \%$. For the instances which could not be solved to optimality, Gap2 is greater than Gap1. This is because we used the optimal solution values obtained from Table 2 to compute Gap1 for these instances, whereas the best feasible solution values were used in the computation of Gap2.

When we also use the extended $F$-partition inequalities, optimal solutions could be found for all instances. Gap1 decreased as well. The average Gap1 reduced to $0.22 \%$ and 2 more instances could be solved at the root node. This shows that extended $F$-partition inequalities are important for the success of the branch-and-cut algorithm. For a few instances, the CPU time increased slightly, but for most of the instances, especially for the instances with $\alpha \in\{3,5\}$, improvement in solution time is significant.

Adding the star-path inequalities, we can say that the performance of the algorithm gets better on average. Although there are a few instances on which the algorithm performs worse, the improvements are more significant. The average Gap1 reduced to $0.19 \%$. We can see that optimal solutions are found for all of the instances in less than $21 \mathrm{~min}$. Without $F$-partition and star-path inequalities, 651 branch-and-cut nodes are necessary to reach the optimal solution on average 
TABLE 1. Results with only clique and cut inequalities.

\begin{tabular}{|c|c|c|c|c|c|c|c|c|}
\hline Instance & $\alpha$ & Opt & Gap1 & Gap2 & NClq & NCut & $\mathrm{Cpu}$ & NNode \\
\hline kroA150 & 3 & 79,572 & 0.85 & 0 & 170 & 1,131 & 111 & 1,109 \\
\hline kroA150 & 5 & 125,435 & 0.08 & 0 & 224 & 320 & 1 & 5 \\
\hline kroA150 & 7 & 140,961 & 0.25 & 1.2 & 718 & 3,652 & * & 2,641 \\
\hline kroA150 & 9 & 113,080 & 0 & 0 & 1,866 & 4,085 & 231 & 1 \\
\hline kroB150 & 3 & 78,180 & 1.26 & 9.18 & 1,112 & 5,014 & * & 2,856 \\
\hline kroB150 & 5 & 122,875 & 1.19 & 2.28 & 1,959 & 170,593 & $*$ & 2,832 \\
\hline kroB150 & 7 & 135,382 & 0 & 0 & 690 & 2,002 & 10 & 1 \\
\hline kroB150 & 9 & 108,885 & 0 & 0 & 1,906 & 4,229 & 227 & 1 \\
\hline u159 & 3 & 126,240 & 0.37 & 0 & 52 & 116 & 11 & 69 \\
\hline u159 & 5 & 204,250 & 0.54 & 0 & 306 & 3,939 & 50 & 113 \\
\hline u159 & 7 & 235,221 & 0 & 0 & 724 & 2,993 & 17 & 1 \\
\hline u159 & 9 & 199,552 & 0 & 0 & 2038 & 5,161 & 281 & 1 \\
\hline rat195 & 3 & 6,957 & 0.85 & 13.97 & 626 & 2,047 & * & 1,692 \\
\hline rat195 & 5 & 11,320 & 0.3 & 0 & 608 & 6,640 & 168 & 523 \\
\hline rat195 & 7 & 12,319 & 0.02 & 0 & 1,004 & 6,470 & 113 & 3 \\
\hline rat195 & 9 & 8,977 & 0 & 0 & 2,849 & 8749 & 872 & 1 \\
\hline d198 & 3 & 47,340 & 0.43 & 0 & 178 & 1,237 & 387 & 1,943 \\
\hline d198 & 5 & 76,945 & 2.18 & 2.54 & 3,970 & 532,153 & $*$ & 1,629 \\
\hline d198 & 7 & 94,300 & 0.19 & 0 & 1,132 & 9,739 & 465 & 209 \\
\hline d198 & 9 & 96,088 & 0 & 0 & 2,864 & 12,565 & 1,099 & 1 \\
\hline kroA200 & 3 & 87,951 & 0.86 & 23.27 & 1,052 & 3,740 & $*$ & 1,606 \\
\hline kroA200 & 5 & 138,885 & 0.53 & 6.15 & 1,793 & 133,500 & * & 1,593 \\
\hline kroA200 & 7 & 158,227 & 0.37 & 0 & 1,256 & 35,163 & 2,639 & 185 \\
\hline kroA200 & 9 & 122,594 & 0 & 0 & 2,586 & 7,170 & 761 & 1 \\
\hline kroB200 & 3 & 88,311 & 0.92 & 11.37 & 616 & 2,239 & * & 1,607 \\
\hline kroB200 & 5 & 138,905 & 0.3 & 0 & 449 & 13,661 & 240 & 253 \\
\hline kroB200 & 7 & 15,6638 & 0 & 0 & 886 & 3,210 & 31 & 1 \\
\hline kroB200 & 9 & 124,043 & 0 & 0 & 2,628 & 6,675 & 819 & 1 \\
\hline
\end{tabular}

TABLE 2. Results with the extended $F$-partition inequalities.

\begin{tabular}{|c|c|c|c|c|c|c|c|c|c|}
\hline Instance & $\alpha$ & Opt & Gap1 & NFP & $\mathrm{Cpu}$ & NNode & gapimp & cpuimp & nodeimp \\
\hline kroA 150 & 3 & 79,572 & 0.54 & 427 & 31 & 159 & 36.59 & 72.07 & 85.66 \\
\hline kroA150 & 5 & 125,435 & 0 & 58 & 0 & 1 & 100 & 100 & 80 \\
\hline kroA150 & 7 & 140,961 & 0.24 & 53 & 70 & 19 & 2.59 & $* *$ & 99.28 \\
\hline kroA150 & 9 & 113,080 & 0 & 10 & 266 & 1 & 0 & -15.15 & 0 \\
\hline kroB150 & 3 & 78,180 & 0.83 & 633 & 44 & 331 & 34.01 & $* *$ & 88.41 \\
\hline kroB150 & 5 & 122,875 & 0.72 & 1,415 & 207 & 413 & 39.12 & $* *$ & 85.42 \\
\hline kroB150 & 7 & 135,382 & 0 & 9 & 10 & 1 & 0 & 0 & 0 \\
\hline kroB150 & 9 & 108,885 & 0 & 12 & 211 & 1 & 0 & 7.05 & 0 \\
\hline u159 & 3 & 126,240 & 0.21 & 71 & 2 & 15 & 43.44 & 81.82 & 78.26 \\
\hline u159 & 5 & 204,250 & 0.16 & 547 & 51 & 95 & 69.48 & -2 & 15.93 \\
\hline u159 & 7 & 235,221 & 0 & 10 & 16 & 1 & 0 & 5.88 & 0 \\
\hline u159 & 9 & 199,552 & 0 & 12 & 270 & 1 & 0 & 3.91 & 0 \\
\hline rat195 & 3 & 6,957 & 0.62 & 1,398 & 128 & 191 & 27.12 & $* *$ & 88.71 \\
\hline rat195 & 5 & 11,320 & 0.14 & 162 & 35 & 59 & 52.94 & 79.17 & 88.72 \\
\hline rat195 & 7 & 12,319 & 0 & 6 & 76 & 1 & 100 & 32.74 & 66.67 \\
\hline rat195 & 9 & 8,977 & 0 & 3 & 938 & 1 & 0 & -7.57 & 0 \\
\hline d198 & 3 & 47,340 & 0.28 & 421 & 114 & 397 & 34.8 & 70.54 & 79.57 \\
\hline d198 & 5 & 76,945 & 0.5 & 507 & 83 & 23 & 76.92 & $* *$ & 98.59 \\
\hline d198 & 7 & 94,300 & 0.13 & 51 & 372 & 155 & 33.01 & 20 & 25.84 \\
\hline d198 & 9 & 96,088 & 0 & 36 & 1,095 & 1 & 0 & 0.36 & 0 \\
\hline kroA200 & 3 & 87,951 & 0.59 & 3,970 & 476 & 1,217 & 30.82 & $* *$ & 24.22 \\
\hline kroA200 & 5 & 138,885 & 0.22 & 518 & 194 & 69 & 57.92 & $* *$ & 95.67 \\
\hline kroA200 & 7 & 158,227 & 0.19 & 118 & 305 & 35 & 49.41 & 88.44 & 81.08 \\
\hline kroA200 & 9 & 122,594 & 0 & 8 & 748 & 1 & 0 & 1.71 & 0 \\
\hline kroB200 & 3 & 88,311 & 0.28 & 378 & 48 & 131 & 69.36 & $* *$ & 91.85 \\
\hline kroB200 & 5 & 138,905 & 0.22 & 469 & 136 & 73 & 25.12 & 43.33 & 71.15 \\
\hline kroB200 & 7 & 156,638 & 0 & 7 & 39 & 1 & 0 & -25.81 & 0 \\
\hline kroB200 & 9 & 124,043 & 0 & 11 & 778 & 1 & 0 & 5.01 & 0 \\
\hline
\end{tabular}


TABLE 3. Results with the extended $F$-partition and star-path inequalities.

\begin{tabular}{|c|c|c|c|c|c|c|c|c|c|}
\hline Instance & $\alpha$ & Opt & Gap1 & NSP & $\mathrm{Cpu}$ & NNode & gapimp & cpuimp & nodeimp \\
\hline kroA 150 & 3 & 79,572 & 0.54 & 0 & 31 & 159 & 0 & 0 & 0 \\
\hline kroA 150 & 5 & 125,435 & 0 & 0 & 0 & 1 & 0 & 0 & 0 \\
\hline kroA150 & 7 & 140,961 & 0.24 & 640 & 48 & 17 & 0 & 31.43 & 10.53 \\
\hline kroA 150 & 9 & 113,080 & 0 & 1,128 & 225 & 1 & 0 & 15.41 & 0 \\
\hline kroB150 & 3 & 78,180 & 0.83 & 0 & 44 & 331 & 0 & 0 & 0 \\
\hline kroB150 & 5 & 122,875 & 0.72 & 34 & 136 & 291 & -0.23 & 34.3 & 29.54 \\
\hline kroB150 & 7 & 135,382 & 0 & 515 & 7 & 1 & 0 & 30 & 0 \\
\hline kroB150 & 9 & 108,885 & 0 & 1,874 & 224 & 1 & 0 & -6.16 & 0 \\
\hline u159 & 3 & 126,240 & 0.21 & 0 & 2 & 15 & 0 & 0 & 0 \\
\hline u159 & 5 & 204,250 & 0.15 & 6 & 25 & 31 & 5.95 & 50.98 & 67.37 \\
\hline u159 & 7 & 235,221 & 0 & 482 & 12 & 1 & 0 & 25 & 0 \\
\hline u159 & 9 & 199,552 & 0 & 1,823 & 299 & 1 & 0 & -10.74 & 0 \\
\hline rat195 & 3 & 6,957 & 0.62 & 0 & 128 & 191 & 0 & 0 & 0 \\
\hline rat195 & 5 & 11,320 & 0.14 & 2 & 35 & 59 & 0 & 0 & 0 \\
\hline rat195 & 7 & 12,319 & 0 & 534 & 59 & 1 & 0 & 22.37 & 0 \\
\hline rat195 & 9 & 8,977 & 0 & 181 & 728 & 1 & 0 & 22.39 & 0 \\
\hline d198 & 3 & 47,340 & 0.28 & 0 & 114 & 397 & 0 & 0 & 0 \\
\hline d198 & 5 & 76,945 & 0.08 & 2 & 29 & 13 & 84.5 & 65.06 & 43.48 \\
\hline d198 & 7 & 94,300 & 0.13 & 1,778 & 339 & 123 & 0 & 8.87 & 20.65 \\
\hline d198 & 9 & 96,088 & 0 & 3,197 & 1,258 & 1 & 0 & -14.89 & 0 \\
\hline kroA200 & 3 & 87,951 & 0.59 & 0 & 476 & 1,217 & 0 & 0 & 0 \\
\hline kroA200 & 5 & 138,885 & 0.22 & 4 & 194 & 69 & 0 & 0 & 0 \\
\hline kroA200 & 7 & 158,227 & 0.16 & 668 & 349 & 73 & 16.72 & -14.43 & -108.57 \\
\hline kroA200 & 9 & 122,594 & 0 & 1,023 & 850 & 1 & 0 & -13.64 & 0 \\
\hline kroB200 & 3 & 88,311 & 0.28 & 0 & 47 & 131 & 0 & 2.08 & 0 \\
\hline kroB200 & 5 & 138,905 & 0.22 & 32 & 116 & 83 & 0 & 14.71 & -13.7 \\
\hline kroB200 & 7 & 156,638 & 0 & 448 & 38 & 1 & 0 & 2.56 & 0 \\
\hline kroB200 & 9 & 124,043 & 0 & 556 & 830 & 1 & 0 & -6.68 & 0 \\
\hline
\end{tabular}

TABLE 4. Results without reduction operations.

\begin{tabular}{|c|c|c|c|c|c|c|c|}
\hline Instance & $\alpha$ & Gap1 & $\mathrm{Cpu}$ & NNode & gapimp & cpuimp & nodeimp \\
\hline kroA 150 & 3 & 0.53 & 49 & 297 & -0.71 & 36.7 & 46.5 \\
\hline kroA 150 & 5 & 0 & 1 & 1 & 0 & 100.0 & 0.0 \\
\hline kroA 150 & 7 & 0.24 & 52 & 17 & 0 & 7.7 & 0.0 \\
\hline kroA 150 & 9 & 0 & 230 & 1 & 0 & 2.2 & 0.0 \\
\hline kroB150 & 3 & 0.83 & 54 & 337 & 0 & 18.5 & 1.8 \\
\hline kroB150 & 5 & 0.72 & 203 & 343 & -0.45 & 33.0 & 15.2 \\
\hline kroB150 & 7 & 0.01 & 13 & 3 & 100 & 46.2 & 66.7 \\
\hline kroB150 & 9 & 0 & 226 & 1 & 0 & 0.9 & 0.0 \\
\hline u159 & 3 & 0.2 & 9 & 13 & -3.14 & 77.8 & -15.4 \\
\hline u159 & 5 & 0.17 & 53 & 55 & 9.46 & 52.8 & 43.6 \\
\hline u159 & 7 & 0.01 & 30 & 3 & 100 & 60.0 & 66.7 \\
\hline u159 & 9 & 0 & 299 & 1 & 0 & 0.0 & 0.0 \\
\hline rat195 & 3 & 0.62 & 133 & 191 & 0 & 3.8 & 0.0 \\
\hline rat195 & 5 & 0.11 & 63 & 65 & -23.08 & 44.4 & 9.2 \\
\hline rat195 & 7 & 0 & 57 & 1 & 0 & -3.5 & 0.0 \\
\hline rat195 & 9 & 0 & 728 & 1 & 0 & 0.0 & 0.0 \\
\hline d198 & 3 & 0.28 & 133 & 415 & 0 & 14.3 & 4.3 \\
\hline d198 & 5 & 0.1 & 93 & 49 & 20 & 68.8 & 73.5 \\
\hline d198 & 7 & 0.15 & 253 & 67 & 10.52 & -34.0 & -83.6 \\
\hline d198 & 9 & 0 & 1,255 & 1 & 0 & -0.2 & 0.0 \\
\hline kroA200 & 3 & 0.59 & 602 & 1,207 & 0 & 20.9 & -0.8 \\
\hline kroA200 & 5 & 0.23 & 126 & 55 & 2.22 & -54.0 & -25.5 \\
\hline kroA200 & 7 & 0.17 & 182 & 19 & 7.09 & -91.8 & -284.2 \\
\hline kroA200 & 9 & 0 & 838 & 1 & 0 & -1.4 & 0.0 \\
\hline kroB200 & 3 & 0.28 & 67 & 195 & -2.88 & 29.9 & 32.8 \\
\hline kroB200 & 5 & 0.22 & 81 & 59 & 0 & -43.2 & -40.7 \\
\hline kroB200 & 7 & 0 & 31 & 1 & 0 & -22.6 & 0.0 \\
\hline kroB200 & 9 & 0 & 830 & 1 & 0 & 0.0 & 0.0 \\
\hline
\end{tabular}


TABLE 5. Results of larger instances.

\begin{tabular}{|c|c|c|c|c|c|c|c|c|c|}
\hline Instance & $\alpha$ & Opt & Gap1 & NClq & NCut & NFP & NSP & $\mathrm{Cpu}$ & Nnode \\
\hline pr226 & 3 & 241,107 & 0.04 & 70 & 209 & 131 & 0 & 5 & 5 \\
\hline pr226 & 5 & 383,055 & 0.69 & 429 & 762 & 192 & 9 & 10 & 5 \\
\hline pr226 & 7 & 469,493 & 0.01 & 981 & 4,393 & 12 & 743 & 85 & 3 \\
\hline pr226 & 9 & 470,711 & 0 & 2,638 & 7,537 & 17 & 1,254 & 596 & 1 \\
\hline gr229 & 3 & 401,445 & 0.28 & 92 & 597 & 732 & 0 & 121 & 259 \\
\hline gr229 & 5 & 622,905 & 0.07 & 382 & 2,617 & 189 & 7 & 44 & 11 \\
\hline gr229 & 7 & 680,052 & 0.02 & 1,084 & 4,620 & 22 & 154 & 121 & 9 \\
\hline gr229 & 9 & 509,687 & 0 & 3,148 & 8,848 & 11 & 793 & 1,756 & 1 \\
\hline gil262 & 3 & 7,116 & 0.2 & 118 & 370 & 319 & 0 & 53 & 111 \\
\hline gil262 & 5 & 11,235 & 0.07 & 454 & 982 & 211 & 3 & 22 & 13 \\
\hline gil262 & 7 & 12,497 & 0 & 1,279 & 8,125 & 14 & 742 & 139 & 1 \\
\hline gil262 & 9 & 9,749 & 0 & 3,958 & 12,226 & 11 & 1,794 & 3,764 & 1 \\
\hline $\operatorname{lin} 318$ & 3 & 126,087 & 0.17 & 182 & 400 & 277 & 0 & 75 & 49 \\
\hline $\operatorname{lin} 318$ & 5 & 202,140 & 0.15 & 618 & 16,769 & 957 & 24 & 490 & 87 \\
\hline $\operatorname{lin} 318$ & 7 & 229,449 & 0 & 1,545 & 9,973 & 9 & 660 & 340 & 1 \\
\hline $\operatorname{lin} 318$ & 9 & 177,089 & 0 & 4,547 & 18,835 & 7 & 411 & 6,299 & 1 \\
\hline
\end{tabular}

while only 115 nodes are required if $F$-partition and star-path inequalities are used.

When we compare the results obtained using the extended $F$-partition and the star-path inequalities together with the results obtained by only using the extended $F$-partition inequalities, there is a slight improvement on average in terms of the number of nodes and the solution time. We also observe that violated extended $F$-partition inequalities can be found for all values of $\alpha$ while violated star-path inequalities could

TABLE 6. Results without heuristic cut separation.

\begin{tabular}{llrrr}
\hline Instance & $\alpha$ & Cpu & NNode & cpuimp \\
\hline kroA150 & 3 & 65 & 495 & 110 \\
kroA150 & 5 & 1 & 1 & 0 \\
kroA150 & 7 & 67 & 17 & 40 \\
kroA150 & 9 & 254 & 1 & 13 \\
kroB150 & 3 & 141 & 915 & 220 \\
kroB150 & 5 & 409 & 363 & 201 \\
kroB150 & 7 & 11 & 1 & 22 \\
kroB150 & 9 & 231 & 1 & 3 \\
u159 & 3 & 3 & 21 & 50 \\
u159 & 5 & 27 & 5 & 8 \\
u159 & 7 & 19 & 1 & 58 \\
u159 & 9 & 306 & 1 & 2 \\
rat195 & 3 & 823 & 2,765 & 543 \\
rat195 & 5 & 76 & 55 & 117 \\
rat195 & 7 & 75 & 1 & 27 \\
rat195 & 9 & 928 & 1 & 27 \\
d198 & 3 & 1,397 & 3,235 & 1,125 \\
d198 & 5 & 319 & 73 & 1,000 \\
d198 & 7 & 443 & 43 & 31 \\
d198 & 9 & 1,295 & 1 & 3 \\
kroA200 & 3 & 642 & 1,165 & 35 \\
kroA200 & 5 & 315 & 73 & 62 \\
kroA200 & 7 & 548 & 53 & 57 \\
kroA200 & 9 & 860 & 1 & 1 \\
kroB200 & 3 & 425 & 1,047 & 804 \\
kroB200 & 5 & 242 & 1 & 34 \\
kroB200 & 7 & 51 & 1 & \\
kroB200 & 9 & 884 & & \\
\hline & & & & \\
\hline
\end{tabular}

not be found for $\alpha=3$. This is consistent with our theoretical findings as most of the nodes are selected as hubs when $\alpha=3$. The number of the star-path inequalities is also small for $\alpha=5$. They are mainly violated when $\alpha \in\{7,9\}$. But for such cases, the optimal solution is found at the root node, so the effect of the star-path inequalities is not as much as the effect of the extended $F$-partition inequalities.

The results obtained by not using the reduction operations are given in Table 4. It is seen that the solution times improved $13 \%$ on average with reduction. In 17 instances the solution time improved, whereas in 8 instances we have longer solution times. It can also be seen that the reduction operations are more effective for $\alpha \in\{3,5\}$.

In Table 5, we provide the results of some larger instances. From this table, it can be seen that instances with up to 318 nodes could be solved to optimality in less than 2 hours.

Finally, in Table 6, we provide the computational results in which the heuristic phase of cut inequality (10) separation is not used. Looking at the solution times, it can be seen that the CPU times increase significantly. The CPU time is $164 \%$ higher on average if the heuristic part is not used. Moreover, for some instances, the CPU times are about 11 times larger without the heuristic cut separation. Hence, our heuristic separation for the cut inequalities seems to be very efficient.

\section{CONCLUSIONS}

In this article, we studied 2ECSSP, which is a special case of a survivable hierarchical telecommunications network design problem. We presented some valid inequalities for the associated polytope and described the conditions for them to be facet defining. The separation problems for the valid inequalities and some reduction operations are discussed. We proposed some heuristic algorithms for separation problems. Based on these findings, a branch-and-cut algorithm is developed.

Our computational analysis showed that the extended $F$-partition and star-path inequalities are important for the 
success of the branch-and-cut algorithm. It is also observed that the heuristic algorithms used for separation problems and the reduction operations are effective in the branch-andcut algorithm. Our experiments showed that the number of branch-and-cut nodes is higher when $\alpha$ is smaller, but it also takes less time to find the optimal solution in this case.

In this article, we only studied survivability of the backbone network in a two level telecommunications network. It may be interesting to incorporate survivability for local access networks. Another interesting extension may be to include the installation of capacities for routing the demands in the network in addition to the survivability aspect.

\section{APPENDIX}

\section{Proof of Theorem 5}

Let $e=\{i, j\} \in E$ with $i \neq 0$ and $j \neq 0$. Suppose that the inequality $\alpha_{e} x_{e}+\beta y \leq \beta_{0}$ is a nontrivial facet defining inequality for $\mathcal{P}_{\mathcal{S}}$ and that conditions (i)-(iv) are satisfied. Define $\mathcal{F}_{\mathcal{S}}=\left\{(x, y) \in P_{S}: \alpha_{e} x_{e}+\beta y=\beta_{0}\right\}$ and $\mathcal{F}=$ $\left\{(x, y) \in P: \alpha_{e} x_{e}+\beta y=\beta_{0}\right\}$. Suppose that all solutions $(x, y) \in \mathcal{F}$ also satisfy $a x+b y=b_{0}$. We will show that $a x+b y=b_{0}$ is a multiple of $\alpha_{e} x_{e}+\beta y=\beta_{0}$. Let $e^{\prime}=$ $\{m, l\} \in E \backslash\{e\}$. There exists a solution $(x, y) \in \mathcal{F}_{\mathcal{S}}$ such that $x_{e^{\prime}}=1$. Let $V^{\prime}=\left\{v \in V: \sum_{k \in V \backslash\{v\}} y_{v k}=0\right\} \cup\{0\}$, i.e., $V^{\prime}$ is the set of backbone nodes.

Suppose that $m \in V \backslash\{0, i, j\}$ and $l \in V \backslash\{0, i, j, m\}$. As $x_{e^{\prime}}=1$, we know that $\left|V^{\prime}\right| \geq 3$. If $\left|V^{\prime}\right|=3$, then $V^{\prime}=\{0, m, l\}$. By (i), there exists $k \in V \backslash\{0, m, l\}$ such that $\beta_{k m}=\beta_{k l}=\beta_{k 0}=0$. The solution $\left(x^{\prime}, y^{\prime}\right)$ with $x^{\prime}=x+\sum_{e^{\prime \prime} \in E\left(V^{\prime} \cup\{k\}\right)}\left(1-x_{e^{\prime \prime}}\right) \chi_{e^{\prime \prime}}$ and $y^{\prime}=y-y_{k m} \gamma_{k m}-$ $y_{k l} \gamma_{k l}-y_{k 0} \gamma_{k 0}$ is in $\mathcal{F}$. Also the solution $\left(x^{\prime}-\chi_{e^{\prime}}, y^{\prime}\right)$ is in $\mathcal{F}$. Hence $a_{e^{\prime}}=0$. If $\left|V^{\prime}\right| \geq 4$ then both solutions $\left(x^{\prime}, y\right)$ with $x^{\prime}=x+\sum_{e^{\prime \prime} \in E\left(V^{\prime}\right) \backslash\{e\}}\left(1-x_{e^{\prime \prime}}\right) \chi_{e^{\prime \prime}}$ and $\left(x^{\prime}-\chi_{e^{\prime}}, y\right)$ are in $\mathcal{F}$ implying that $a_{e^{\prime}}=0$.

Suppose that $m \in V \backslash\{0, i, j\}$ and $l=i$ or $l=j$. Assume that $l=i$. Again, we know that $\left|V^{\prime}\right| \geq 3$ and if $\left|V^{\prime}\right|=3$ then $V^{\prime}=\{0, i, m\}$. By (ii), there exists $k \in V \backslash\{0, m, i, j\}$ such that $\beta_{k m}=\beta_{k i}=\beta_{k 0}=0$. Let $x^{\prime}=x+\sum_{e^{\prime \prime} \in E\left(V^{\prime} \cup\{k\}\right)}\left(1-x_{e^{\prime \prime}}\right) \chi_{e^{\prime \prime}}$ and $y^{\prime}=y-y_{k m} \gamma_{k m}-y_{k i} \gamma_{k i}-y_{k 0} \gamma_{k 0}$. As both solutions $\left(x^{\prime}, y^{\prime}\right)$ and $\left(x^{\prime}-\chi_{e^{\prime}}, y^{\prime}\right)$ are in $\mathcal{F}$ we can conclude that $a_{e^{\prime}}=0$. If $\left|V^{\prime}\right|=4$ and $V^{\prime}=\{0, m, i, v\}$ for some $v \in V \backslash\{0, m, i, j\}$ or $\left|V^{\prime}\right| \geq 5$ then both solutions $\left(x^{\prime}, y\right)$ with $x^{\prime}=x+\sum_{e^{\prime \prime} \in E\left(V^{\prime}\right) \backslash\{e\}}\left(1-x_{e^{\prime \prime}}\right) \chi_{e^{\prime \prime}}$ and $\left(x^{\prime}-\chi_{e^{\prime}}, y\right)$ are in $\mathcal{F}$. Hence $a_{e^{\prime}}=0$. The only remaining case is $V^{\prime}=\{0, m, i, j\}$. By (ii), there exists $k \in V \backslash\{0, m, i, j\}$ such that $\beta_{k m}=\beta_{k i}=$ $\beta_{k 0}=\beta_{k j}=0$. Let $x^{\prime}=x+\sum_{e^{\prime \prime} \in E\left(V^{\prime} \cup\{k\}\right) \backslash\{e\}}\left(1-x_{e^{\prime \prime}}\right) \chi_{e^{\prime \prime}}$ and $y^{\prime}=y-y_{k m} \gamma_{k m}-y_{k i} \gamma_{k i}-y_{k j} \gamma_{k j}-y_{k 0} \gamma_{k 0}$. As $\left(x^{\prime}, y^{\prime}\right)$ and $\left(x^{\prime}-\chi e^{\prime}, y^{\prime}\right)$ are both in $\mathcal{F}$, we have $a_{e^{\prime}}=0$. The case with $l=j$ is the same.

Suppose that $e^{\prime}=\{0, m\}$ with $m \in V \backslash\{0, i, j\}$. This time, we know that $\left|V^{\prime}\right| \geq 2$. If $\left|V^{\prime}\right|=2$, then $V^{\prime}=\{0, m\}$. Condition (iii) implies that there exist two distinct nodes $k_{1}, k_{2} \in V \backslash\{0, m\}$ such that $\beta_{k_{1} m}=\beta_{k_{1} 0}=\beta_{k_{2} m}=$ $\beta_{k_{2} 0}=0$. Consider the solution $\left(x^{\prime}, y^{\prime}\right)$ with $x^{\prime}=x+$ $\sum_{e^{\prime \prime} \in E\left(V^{\prime} \cup\left\{k_{1}, k_{2}\right\}\right) \backslash\{e\}}\left(1-x_{e^{\prime \prime}}\right) \chi_{e^{\prime \prime}}$ and $y^{\prime}=y-y_{k_{1} m} \gamma_{k_{1} m}-$ $y_{k_{1} 0} \gamma_{k_{1} 0}-y_{k_{2} m} \gamma_{k_{2} m}-y_{k_{2} 0} \gamma_{k_{2} 0}$. As $\left(x^{\prime}, y^{\prime}\right)$ and $\left(x^{\prime}-\chi_{e^{\prime}}, y^{\prime}\right)$ are in $\mathcal{F}$ we can conclude that $a_{e^{\prime}}=0$. If $\left|V^{\prime}\right|=3$ and $V^{\prime}=\{0, m, v\}$ for some $v \in V \backslash\{0, m, i, j\}$, then by (i), we know that there exists $k \in V \backslash\{0, m, v\}$ such that $\beta_{k m}=\beta_{k v}=\beta_{k 0}=0$. Let $x^{\prime}=x+\sum_{e^{\prime \prime} \in E\left(V^{\prime} \cup\{k\}\right)}\left(1-x_{e^{\prime \prime}}\right) \chi_{e^{\prime \prime}}$ and $y^{\prime}=y-y_{k m} \gamma_{k m}-$ $y_{k v} \gamma_{k v}-y_{k 0} \gamma_{k 0}$. Then as $\left(x^{\prime}, y^{\prime}\right)$ and $\left(x^{\prime}-\chi_{e^{\prime}}, y^{\prime}\right)$ are both in $\mathcal{F}$, we have $a_{e^{\prime}}=0$. If $\left|V^{\prime}\right|=3$ and $V^{\prime}=\{0, m, v\}$ for some $v \in\{i, j\}$, say without loss of generality that $v=i$, then by (ii), we know that there exists $k \in V \backslash\{0, m, i, j\}$ such that $\beta_{k m}=\beta_{k i}=\beta_{k 0}=0$. Let $x^{\prime}=x+\sum_{e^{\prime \prime} \in E\left(V^{\prime} \cup\{k\}\right)}\left(1-x_{e^{\prime \prime}}\right) \chi_{e^{\prime \prime}}$ and $y^{\prime}=y-y_{k m} \gamma_{k m}-y_{k i} \gamma_{k i}-y_{k 0} \gamma_{k 0}$. As both solutions $\left(x^{\prime}, y^{\prime}\right)$ and $\left(x^{\prime}-\chi_{e^{\prime}}, y^{\prime}\right)$ are in $\mathcal{F}$, we can conclude that $a_{e^{\prime}}=0$. If $\left|V^{\prime}\right| \geq 4$, then $\left(x^{\prime}, y\right)$ with $x^{\prime}=x+\sum_{e^{\prime \prime} \in E\left(V^{\prime}\right) \backslash\{e\}}\left(1-x_{e^{\prime \prime}}\right) \chi_{e^{\prime \prime}}$ and $\left(x^{\prime}-\chi_{e^{\prime}}, y\right)$ are in $\mathcal{F}$. Hence $a_{e^{\prime}}=0$.

Suppose that $e^{\prime}=\{0, i\}$. If $\left|V^{\prime}\right|=2$, then $V^{\prime}=\{0, i\}$. By (iv), there exist $k_{1}, k_{2} \in V \backslash\{0, i, j\}$ such that $\beta_{k_{1} i}=$ $\beta_{k_{1} 0}=\beta_{k_{2} i}=\beta_{k_{2} 0}=0$. Both solutions $\left(x^{\prime}, y^{\prime}\right)$ with $x^{\prime}=x+\sum_{e^{\prime \prime} \in E\left(V^{\prime} \cup\left\{k_{1}, k_{2}\right\}\right)}\left(1-x_{e^{\prime \prime}}\right) \chi_{e^{\prime \prime}}$ and $y^{\prime}=y-y_{k_{1} i} \gamma_{k_{1} i}-$ $y_{k_{1} 0} \gamma_{k_{1} 0}-y_{k_{2} i} \gamma_{k_{2} i}-y_{k_{2} 0} \gamma_{k_{2} 0}$ and $\left(x^{\prime}-\chi_{e^{\prime}}, y^{\prime}\right)$ are in $\mathcal{F}$. Thus we have $a_{e^{\prime}}=0$. If $\left|V^{\prime}\right|=3$ and $V^{\prime}=\{0, i, v\}$ for some $v \neq j$ then by (ii), there exists $k \in V \backslash\{0, i, v, j\}$ such that $\beta_{k i}=\beta_{k v}=\beta_{k 0}=0$. Let $x^{\prime}=x+\sum_{e^{\prime \prime} \in E\left(V^{\prime} \cup\{k\}\right)}\left(1-x_{e^{\prime \prime}}\right) \chi_{e^{\prime \prime}}$ and $y^{\prime}=y-y_{k i} \gamma_{k m}-y_{k v} \gamma_{k v}-y_{k 0} \gamma_{k 0}$. Then both solutions $\left(x^{\prime}, y^{\prime}\right)$ and $\left(x^{\prime}-\chi_{e^{\prime}}, y^{\prime}\right)$ are in $\mathcal{F}$. So $a_{e^{\prime}}=0$. If $\left|V^{\prime}\right|=3$ and $V^{\prime}=\{0, i, j\}$, then by (iv), there exist $k_{1}, k_{2} \in V \backslash\{0, i, j\}$ such that $\beta_{k_{1} i}=\beta_{k_{1} 0}=\beta_{k_{1} j}=\beta_{k_{2} i}=\beta_{k_{2} 0}=\beta_{k_{2} j}=0$. Let $x^{\prime}=x+\sum_{e^{\prime \prime} \in E\left(V^{\prime} \cup\left\{k_{1}, k_{2}\right\}\right) \backslash\{e\}}\left(1-x_{e^{\prime \prime}}\right) \chi_{e^{\prime \prime}}$ and $y^{\prime}=$ $y-y_{k_{1} i} \gamma_{k_{1} i}-y_{k_{1} j} \gamma_{k_{1} j}-y_{k_{1}} 0 \gamma_{k_{1}} 0-y_{k_{2} i} \gamma_{k_{2} i}-y_{k_{2} j} \gamma_{k_{2} j}-y_{k_{2}} 0 \gamma_{k_{2}} 0$. As the solutions $\left(x^{\prime}, y^{\prime}\right)$ and $\left(x^{\prime}-\chi_{e^{\prime}}, y^{\prime}\right)$ are in $\mathcal{F}, a_{e^{\prime}}=0$. If $\left|V^{\prime}\right|=4$ and $V^{\prime}=\{0, i, j, v\}$, then by (ii), there exists $k \in V \backslash\{0, i, v, j\}$ such that $\beta_{k i}=\beta_{k v}=\beta_{k j}=\beta_{k 0}=0$. Let $x^{\prime}=x+\sum_{e^{\prime \prime} \in E\left(V^{\prime} \cup\{k\}\right) \backslash\{e\}}\left(1-x_{e^{\prime \prime}}\right) \chi_{e^{\prime \prime}}$ and $y^{\prime}=y-y_{k i} \gamma_{k i}-$ $y_{k v} \gamma_{k v}-y_{k j} \gamma_{k j}-y_{k 0} \gamma_{k 0}$. Both solutions $\left(x^{\prime}, y^{\prime}\right)$ and $\left(x^{\prime}-\chi_{e^{\prime}}, y^{\prime}\right)$ are in $\mathcal{F}$. So $a_{e^{\prime}}=0$. If $\left|V^{\prime}\right|=4$ and $j \notin V^{\prime}$ or if $\left|V^{\prime}\right| \geq 5$, then solutions $\left(x^{\prime}, y\right)$ with $x^{\prime}=x+\sum_{e^{\prime \prime} \in E\left(V^{\prime}\right) \backslash\{e\}}\left(1-x_{e^{\prime \prime}}\right) \chi_{e^{\prime \prime}}$ and $\left(x^{\prime}-\chi_{e^{\prime}}, y\right)$ are in $\mathcal{F}$. Hence, $a_{e^{\prime}}=0$.

We proved that $a x+b y=b_{0}$ is equal to $a_{e} x_{e}+b y=b_{0}$.

Let $(m, l) \in A$ with $\beta_{m l}=0$ and $(x, y) \in \mathcal{F}_{\mathcal{S}}$ be such that $y_{m l}=1$. Let $V^{\prime}=\left\{v \in V: \sum_{k \in V \backslash\{v\}} y_{v k}=0\right\} \cup\{0\}$. If $\left|V^{\prime}\right|=1$, then $V^{\prime}=\{0\}$ and $l=0$. If $m \neq i, j$ then by (iii), there exist two distinct nodes $k_{1}, k_{2} \in V \backslash(\{0, m\})$ such that $\left|\left\{k_{1}, k_{2}\right\} \cap\{i, j\}\right| \leq 1$ and $\beta_{k_{1} 0}=\beta_{k_{2} 0}=0$. If $m=i$ or $m=j$, then by (iv), there exist two distinct nodes $k_{1}, k_{2} \in V \backslash(\{0, i, j\})$ such that $\beta_{k_{1} 0}=\beta_{k_{2} 0}=0$. In both cases, the solution $\left(x^{\prime}, y^{\prime}\right)$ where $x^{\prime}=x+\sum_{e^{\prime} \in E\left(\left\{0, k_{1}, k_{2}\right\}\right)}\left(1-x_{e^{\prime}}\right) \chi_{e^{\prime}}$ and $y^{\prime}=y-y_{k_{1} 0} \gamma_{k_{1} 0}-y_{k_{2} 0 \gamma_{k_{2} 0}}$ is in $\mathcal{F}$. Also the solution $\left(x^{\prime}+\sum_{e^{\prime} \in E\left(\left\{0, k_{1}, k_{2}, m\right\}\right)} \chi_{e^{\prime}}, y^{\prime}-\gamma_{m 0}\right)$ is in $\mathcal{F}$. Hence, we have $b_{m 0}=0$. If $\left|V^{\prime}\right|=2$, then suppose $V^{\prime}=\{0, v\}$ for some $v \in V \backslash\{0, m\}$ such that $l \in V^{\prime}$. If $v \neq i, j$, then by (i), there exists a node $k \in V \backslash\{0, m, v\}$ such that $\beta_{k 0}=\beta_{k v}=0$. If $v \in\{i, j\}$, then by (ii), there exists a node $k \in V \backslash\{0, m, i, j\}$ such that $\beta_{k 0}=\beta_{k v}=0$. The solution $\left(x^{\prime}, y^{\prime}\right)$ where $x^{\prime}=$ $x+\sum_{e^{\prime} \in E(\{0, k, v\})}\left(1-x_{e^{\prime}}\right) \chi_{e^{\prime}}$ and $y^{\prime}=y-y_{k 0} \gamma_{k 0}-y_{k v} \gamma_{k v}$ is in $\mathcal{F}$. As the solution $\left(x+\sum_{e^{\prime} \in E(\{0, k, v, m\}) \backslash\{e\}} \chi_{e^{\prime}}, y^{\prime}-\gamma_{m l}\right)$ is also in $\mathcal{F}$, we have $b_{m l}=0$. If $\left|V^{\prime}\right|=3$ and $V^{\prime}=\{0, i, j\}$, then by (ii), there exists a node $k \in V \backslash\{0, m, i, j\}$ such that $\beta_{k 0}=$ $\beta_{k i}=\beta_{k j}=0$. Let $x^{\prime}=x+\sum_{e^{\prime} \in E(\{0, i, j, k\}) \backslash\{e\}}\left(1-x_{e^{\prime}}\right) \chi_{e^{\prime}}$ and 
$y^{\prime}=y-y_{k 0} \gamma_{k 0}-y_{k i} \gamma_{k i}-y_{k j} \gamma_{k j}$. Both solutions $\left(x^{\prime}, y^{\prime}\right)$ and $\left(x+\sum_{e^{\prime} \in E(\{0, i, j, k, m\}) \backslash\{e\}} \chi_{e^{\prime}}, y^{\prime}-\gamma_{m l}\right)$ are in $\mathcal{F}$, so we have $b_{m l}=0$. Finally, if $\left|V^{\prime}\right|=3$ and $\left|\{i, j\} \cap V^{\prime}\right| \leq 1$ or $\left|V^{\prime}\right| \geq 4$, then $\left(x^{\prime}, y\right)$ and $\left(x^{\prime}+\sum_{e^{\prime} \in E\left(V^{\prime} \cup\{m\}\right) \backslash\{e\}} \chi_{e^{\prime}}, y-\gamma_{m l}\right)$ are both in $\mathcal{F}$, where $x^{\prime}=x+\sum_{e^{\prime} \in E\left(V^{\prime}\right) \backslash\{e\}}\left(1-x_{e^{\prime}}\right) \chi_{e^{\prime}}$. So we can conclude that $b_{m l}=0$. Hence, $b_{m l}=0$ for all $(m, l) \in A$ with $\beta_{m l}=0$.

Now assume that there exists $(x, y) \in \mathcal{F}_{\mathcal{S}}$ such that $a_{e} x_{e}+$ by $\neq b_{0}$. Let $V^{\prime}=\left\{v \in V: \sum_{k \in V \backslash\{v\}} y_{v k}=0\right\} \cup\{0\}$. Unless $\left|V^{\prime}\right|=2$ or $V^{\prime}=\{0, i, j\}$ and $x_{e}=0$, the solution $\left(x^{\prime}, y\right)$ where $x^{\prime}=x+\sum_{e^{\prime} \in E\left(V^{\prime}\right) \backslash\{e\}}\left(1-x_{e^{\prime}}\right) \chi_{e^{\prime}}$ is in $\mathcal{F}$ and $a_{e} x_{e}^{\prime}+b y \neq b_{0}$. Hence, either $\left|V^{\prime}\right|=2$ or $V^{\prime}=\{0, i, j\}$ and $x_{e}=0$. First suppose that $\left|V^{\prime}\right|=2$ and that $V^{\prime}=\{0, m\}$. If $m \neq i, j$, then by (iii), there exists a node $k \in V \backslash\{0, m\}$ such that $\beta_{k m}=\beta_{k 0}=0$. If $m \in\{i, j\}$, then by (iv), there exists a node $k \in V \backslash\{0, i, j\}$ such that $\beta_{k m}=\beta_{k 0}=0$. In both cases, the solution $\left(x^{\prime}, y^{\prime}\right)$ where $x^{\prime}=x+\sum_{e^{\prime} \in E(\{0, m, k\})}\left(1-x_{e^{\prime}}\right) \chi_{e^{\prime}}$ and $y^{\prime}=y-y_{k 0} \gamma_{k 0}-y_{k m} \gamma_{k m}$ is in $\mathcal{F}$ and $a_{e} x_{e}^{\prime}+b y^{\prime} \neq b_{0}$. Now suppose that $V^{\prime}=\{0, i, j\}$ and $x_{e}=0$. By (iv), there exists a node $k \in V \backslash\{0, i, j\}$ such that $\beta_{k i}=\beta_{k j}=\beta_{k 0}=0$. Now the solution $\left(x^{\prime}, y^{\prime}\right)$ where $x^{\prime}=x+\sum_{e^{\prime} \in E(\{0, i, j, k\}) \backslash\{e\}}\left(1-x_{e^{\prime}}\right) \chi_{e^{\prime}}$ and $y^{\prime}=y-y_{k 0} \gamma_{k 0}-y_{k i} \gamma_{k i}-y_{k j} \gamma_{k j}$ is in $\mathcal{F}$ and $a_{e} x_{e}^{\prime}+b y^{\prime} \neq$ $b_{0}$, a contradiction. So we can conclude that all solutions $(x, y) \in \mathcal{F}_{\mathcal{S}}$ satisfy $a_{e} x_{e}+b y=b_{0}$. Hence, $a_{e} x_{e}+b y=b_{0}$ is a multiple of $\alpha_{e} x_{e}+\beta y=\beta_{0}$.

\section{Acknowledgments}

The authors thank the anonymous referee for his/her comments that permitted us to considerably improve the presentation of this article.

\section{REFERENCES}

[1] E. Balas and M.W. Padberg, Set partitioning: A survey, SIAM Rev 18 (1976), 710-760.

[2] F. Barahona and A.R. Mahjoub, On two-connected subgraph polytopes, Discrete Math 147 (1995), 19-34.

[3] F. Bendali, I. Diarrasouba, A.R. Mahjoub, M. Didi Biha, and J. Mailfert, A branch-and-cut algorithm for the $k$-edge connected subgraph problem, Networks 55 (2010), 13-32.

[4] P. Chardaire, J.L. Lutton, and A. Sutter, Upper and lower bounds for the two-level simple plant location problem, Ann of Oper Res 86 (1999), 117-140.

[5] J. Current and H. Pirkul, The hierarchical network design problem with transshipment facilities, Euro J Oper Res 52 (1991), 338-347.

[6] G. Dahl, D. Huygens, A.R. Mahjoub, and P. Pesneau, On the $k$ edge-disjoint 2-hop-constrained paths polytope, Oper Res Lett 34 (2006), 577-582.

[7] J. Fonlupt and A.R. Mahjoub, Critical extreme points of the 2-edge connected spanning subraph problem, Math Program B 105 (2006), 289-310.

[8] B. Fortz and M. Labbé, Polyhedral results for two-connected networks with bounded rings, Math Program 93 (2002), 27-54.

[9] B. Fortz, M. Labbé, and F. Maffioli, Solving the twoconnected network with bounded meshes problem, Oper Res 58 (2000), 866-877.
[10] B. Fortz, A.R. Mahjoub, S.T. McCormick, and P. Pesneau, Two-edge connected subgraphs with bounded rings: Polyhedral results and branch-and-cut, Math Program 105 (2006), 85-111.

[11] B. Gavish, Topological design of centralized computer networks: Formulations and algorithms, Networks 12 (1982), 355-377.

[12] E. Gourdin, M. Labbé, and H. Yaman, "Telecommunication and location," Facility location: Applications and theory, Chapter 9, Z. Drezner and H.W. Hamacher (Editors), Springer, Berlin, 2001, pp. 275-305.

[13] A.V. Goldberg and R.E. Tarjan, A new approach to the maximum flow problem, J Assoc Comput Mach 35 (1988), 931-940.

[14] M. Grötschel, C.L. Monma, and M. Stoer, "Design of survivable networks," Handbooks in operations research and management Science, M.O. Ball, T.L. Magnanti, C.L. Monma, and G.L. Nemhauser (Editors), Vol. 7, Elsevier, Amsterdam, 1995, pp. 617-671.

[15] J. Hao and J.B. Orlin, A faster algorithm for finding the minimum cut in a directed graph, J Algorithms 17 (1994), 424-446.

[16] D. Huygens, A.R. Mahjoub, and P. Pesneau, Two edgedisjoint hop-constrained paths and polyhedra, SIAM J Disc Math 18 (2004), 287-312.

[17] H. Kerivin and A.R. Mahjoub, Design of survivable networks: A review, Networks 46 (2005), 1-21.

[18] H. Kerivin and A.R. Mahjoub, On survivable network polyhedra, Disc Math 290 (2005), 183-210.

[19] H. Kerivin, A.R. Mahjoub, and C. Nocq, "(1,2)-Survivable networks: Facets and branch-and-cut," The sharpest-cut, Chapter 9, M. Grötschel (Editor), MPS/SIAM Optimization, Philadelphia, 2004, pp. 121-152.

[20] J.G. Klincewicz, Hub location in backbone/tributary network design: A review, Location Science 6 (1998), 307-335.

[21] M. Labbé, G. Laporte, I.R. Martin, and J.J.S. Gonzalez, The ring star problem: Polyhedral analysis and exact algorithm, Networks 43 (2004), 177-189.

[22] M. Labbé and H. Yaman, Solving the hub location problem in a star-star network, Networks 51 (2008), 19-33.

[23] M. Labbé, H. Yaman, and E. Gourdin, A branch and cut algorithm for hub location problems with single assignment, Math Program 102 (2005), 371-405.

[24] Y. Lee, B.H. Lim, and J.S. Park, A hub location problem in designing digital data service networks: Lagrangian relaxation approach, Location Science 4 (1996), 185-194.

[25] A.R. Mahjoub, Two-edge connected spanning subgraphs and polyhedra, Math Program 64 (1994), 199-208.

[26] A.R. Mahjoub, On perfectly two-edge connected graphs, Disc Math 170 (1997), 153-172.

[27] G.L. Nemhauser, Integer and combinatorial optimization, Wiley, New York, 1998.

[28] H. Pirkul and V. Nagarajan, Locating concentrators in centralized computer networks, Ann Oper Res 36 (1992), 247-262.

[29] G. Reinelt, TSPLIB - a traveling salesman problem library, ORSA J Comp 3 (1991), 376-384.

[30] M. Stoer, "Design of survivable networks," Lecture notes in mathematics, Vol. 1531, Springer, Berlin, 1992.

[31] D. Vandenbussche and G.L. Nemhauser, The 2-edgeconnected subgraph polyhedron, J Comb Opt 9 (2005), 357-379. 